\title{
Collocated finite volume schemes for the simulation of natural convective flows on unstructured meshes
}

\author{
Eric Chénier ${ }^{1}$, Robert Eymard $^{1}$, Raphaèle Herbin $^{2, *}$ and Ouardia Touazi ${ }^{1}$ \\ 1 Université Paris-Est, LETEM, EA 2546, 5 bd Descartes, 77454 Marne la Vallée Cedex 2, France \\ eric.chenier, robert.eymard, ouardia.touazi@univ-mlv.fr \\ 2 Université de Marseille, LATP, UMR 6632, 39 rue Joliot Curie \\ 13453 Marseille 13, France, herbin@cmi.univ-mrs.fr
}

\begin{abstract}
SUMMARY
We describe here a collocated finite volume scheme which was recently developed for the numerical simulation of the incompressible Navier-Stokes equations on unstructured meshes, in 2 or 3 space dimensions. We recall its convergence in the case of the linear Stokes equations, and we prove a convergence theorem for the case of the Navier-Stokes equations under the Boussinesq hypothesis. We then present several numerical studies. A comparison between a cluster-type stabilization technique and the more classical Brezzi-Pitkäranta method is performed, the numerical convergence properties are presented on both analytical solutions and benchmark problems and the scheme is finally applied to the study of the natural convection between two eccentric cylinders. Copyright (c) 2006 John Wiley \& Sons, Ltd.

KEY WORDS: cell centered finite volumes, incompressible Navier-Stokes, Boussinesq hypothesis, stabilization methods, unstructured meshes, natural convection between cylinders
\end{abstract}

\section{Introduction}

Numerical schemes for the incompressible Navier-Stokes equations have been extensively studied: see $[17,19,18,26]$ and references therein. An advantage of the finite volume schemes is that the unknowns are approximated by piecewise constant functions: this makes it easy to take into account additional nonlinear phenomena or the coupling with algebraic or differential equations, for instance in the case of reactive flows; in particular, one can find in [28] the presentation of the classical finite volume scheme on rectangular meshes, which has been the basis of many industrial applications. Proofs of the convergence of the so-called "MAC scheme" [21] were performed for the Stokes and Navier-Stokes equations, see [3] and references therein. However, the use of rectangular grids makes an important limitation to the type of

*Correspondence to: LATP, UMR 6632, Université de Provence, 39 rue Joliot Curie 13453 Marseille 13, France

Raphaele.Herbin@cmi.univ-mrs.fr 
domain which can be gridded and more recently, finite volume schemes for the Navier-Stokes equations on triangular grids have been presented, either staggered [20], or collocated [5] where primal variables are used with a Chorin type projection method to ensure the divergence condition. Since staggered schemes have the reputation of being the most stable schemes for incompressible flows, our idea was to generalize the MAC scheme to triangular meshes. Hence we considered a scheme where the velocity unknowns were associated to the control volumes of the mesh, and the "classical" four points cell-centered scheme [22, 9] was applied to discretize the Laplacian of the velocities, while a Galerkin expansion was introduced for the pressure, with the pressure unknowns associated to the vertices of the mesh. Some interesting stability and convergence properties were obtained for this scheme [10, 11, 4, 12], and [23] for a review. However, we were not able to generalize the scheme to the three-dimensional case. We then developed and studied a collocated scheme [14, 13], where velocities and pressure are all collocated within the control volume.

We present here a generalization of this scheme to the complete problem of the incompressible Navier-Stokes equations coupled with the energy conservation under the Boussinesq hypothesis. As mentioned in the introduction, the unknowns are chosen to be collocated; it is well known that the resulting scheme is non elliptic (see e.g. [2]) unless a stabilization technique is used. In the present paper we study two techniques of stabilization. The first one is a BrezziPitkäranta type stabilization [6], the second one is based on the local redistribution of the fluid mass within clusters of control volumes. Both techniques lead to a modified discrete divergence operator. The discretization of the pressure gradient in the momentum balance equation is then performed to ensure, by construction, that it is the transpose of the discrete divergence term of the mass balance equation without stabilization. Finally, the contribution of the discrete nonlinear advection term to the kinetic energy balance vanishes for the modified discrete divergence free velocity fields, as in the continuous case. These features appear to be essential in the theoretical proof of convergence.

This paper is organized as follows. In section 2, we present the finite volume discretization framework, that we apply to the linear Stokes problem. Then, in section 3, we present the adaptation of the scheme to the nonlinear problem resulting from the steady Navier-Stokes equations under the Boussinesq hypothesis. We then state a convergence theorem for the scheme in this framework. We then discuss and compare the stabilization methods in section 4 on a numerical example, illustrate the convergence properties on both analytical solutions and benchmark problems and present original numerical results obtained in the case of the natural convection between two eccentric cylinders.

2. Principles of the collocated scheme applied to the linear case

In this section, we first give all the tools necessary for the definition of the schemes, followed by their application to the framework of the linear Stokes problem.

\subsection{Admissible meshes, discrete spaces and operators}

Let $\Omega$ be an open bounded polygonal subset or $\mathbb{R}^{d}$. We consider admissible meshes $\mathcal{D}=$ $(\mathcal{M}, \mathcal{E}, \mathcal{P})$ in the sense of [9], that is: $\mathcal{M}$ is a partition of $\Omega$ by polygonal (or polyhedral) convex subsets of $\mathbb{R}^{d}, \mathcal{E}$ is the set of edges of these subsets, and $\mathcal{P}=\left(\boldsymbol{x}_{K}\right)_{K \in \mathcal{M}}$ is a set of 
points satisfying the usual orthogonality condition (see Figure 1). The following notations are

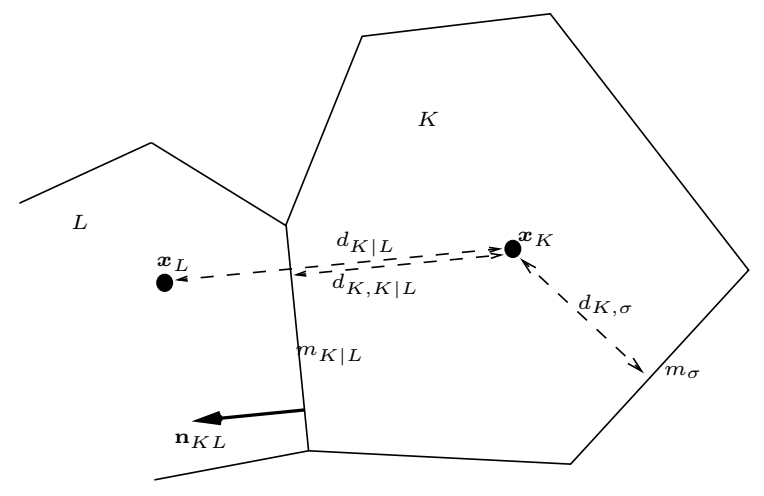

Figure 1. Notations for an admissible mesh

used. The size of the discretization is defined by: $h_{\mathcal{D}}=\sup \{\operatorname{diam}(K), K \in \mathcal{M}\}$. The set of interior (resp. boundary) edges is denoted by $\mathcal{E}_{\text {int }}$ (resp. $\mathcal{E}_{\text {ext }}$ ), that is $\mathcal{E}_{\text {int }}=\{\sigma \in \mathcal{E} ; \sigma \not \subset \partial \Omega\}$ (resp. $\mathcal{E}_{\text {ext }}=\{\sigma \in \mathcal{E} ; \sigma \subset \partial \Omega\}$ ). For any edge $\sigma \in \mathcal{E}$, we denote by $\mathrm{m}_{\sigma}$ the length or area of $\sigma$. For all $K \in \mathcal{M}$ and $\sigma \in \mathcal{E}_{K}$ (the set of edges of $K$ ), we denote by $\boldsymbol{n}_{K, \sigma}$ the unit vector normal to $\sigma$ outward to $K$ and we denote by $d_{K, \sigma}$ the Euclidean distance between $\boldsymbol{x}_{K}$ and $\sigma$. For all $K \in \mathcal{M}$, we denote by $\mathrm{m}_{K}$ the surface or volume of $K, \mathcal{N}_{K}$ the subset of $\mathcal{M}$ of the neighboring control volumes. For all $K \in \mathcal{M}$ and $L \in \mathcal{N}_{K}$, we denote by $K \mid L \in \mathcal{E}_{K} \cap \mathcal{E}_{L}$ the common edge of $K$ and $L$; we then set $\boldsymbol{n}_{K L}=\boldsymbol{n}_{K, K \mid L}$, and we denote by $d_{K \mid L}=d_{K, K \mid L}+d_{L, K \mid L}$ the Euclidean distance between $\boldsymbol{x}_{K}$ and $\boldsymbol{x}_{L}$. We shall measure the regularity of the mesh through the function $\operatorname{regul}(\mathcal{D})$ defined by

$$
\begin{aligned}
\operatorname{regul}(\mathcal{D})=\inf & \left\{\frac{d_{K, \sigma}}{\operatorname{diam}(K)}, K \in \mathcal{M}, \sigma \in \mathcal{E}_{K}\right\} \\
& \cup\left\{\frac{d_{K, K \mid L}}{d_{K \mid L}}, K \in \mathcal{M}, L \in \mathcal{N}_{K}\right\} \cup\left\{\frac{1}{\operatorname{card}\left(\mathcal{E}_{K}\right)}, K \in \mathcal{M}\right\} .
\end{aligned}
$$

We then denote by $H_{\mathcal{D}}(\Omega) \subset L^{2}(\Omega)$ the space of functions which are piecewise constant on each control volume $K \in \mathcal{M}$. For any $u \in H_{\mathcal{D}}(\Omega)$ and for all $K \in \mathcal{M}$, we denote by $u_{K}$ the constant value of $u$ in $K$ and we define the discrete finite volume Laplacian $\Delta_{\mathcal{D}} u \in H_{\mathcal{D}}(\Omega)$ (corresponding to the homogeneous Dirichlet boundary conditions), such that for all $K \in \mathcal{M}$, the constant value $\Delta_{K} u$ of $\Delta_{\mathcal{D}} u$ in $K$ is given by:

$$
\Delta_{K} u=\frac{1}{\mathrm{~m}_{K}}\left(\sum_{L \in \mathcal{N}_{K}} \frac{\mathrm{m}_{K \mid L}}{d_{K \mid L}}\left(u_{L}-u_{K}\right)+\sum_{\sigma \in \mathcal{E}_{K} \cap \mathcal{E}_{\text {ext }}} \frac{\mathrm{m}_{\sigma}}{d_{K, \sigma}}\left(0-u_{K}\right)\right) .
$$

Note that this approximation is indeed obtained by a finite volume principle; in fact, for a continuous function $\bar{u} \in C^{2}(\Omega)$, integrating $\Delta u$ over a control volume $K$ and using the Gauss divergence theorem and decomposing the boundary $\partial K$ of $K$ into its edges $\left(\partial K=\cup_{\sigma \in \mathcal{E}_{K}} \sigma\right)$ yields:

$$
\int_{K} \Delta u(x) \mathrm{d} \boldsymbol{x}=\int_{\partial K} \nabla u(x) \cdot \boldsymbol{n}_{K}(x) \mathrm{d} \gamma(\boldsymbol{x})=\sum_{\sigma \in \mathcal{E}_{K}} \int_{\sigma} \nabla u(x) \cdot \boldsymbol{n}_{K, \sigma} \mathrm{d} \gamma(\boldsymbol{x})
$$


where $\boldsymbol{n}_{K}$ (resp. $\boldsymbol{n}_{K, \sigma}$ ) denotes the outward unit normal vector to $\partial K$ (resp. unit normal vector to $\sigma$ outward to $K$ ) and $\mathrm{d} \gamma$ denotes the integration sign on the boundary. Under the orthogonality condition on the mesh, the quotients $\frac{\bar{u}\left(\boldsymbol{x}_{L}\right)-\bar{u}\left(\boldsymbol{x}_{K}\right)}{d_{K \mid L}}$ and $\frac{0-\bar{u}\left(\boldsymbol{x}_{K}\right)}{d_{K, \sigma}}$ are consistent finite difference approximations of $\nabla u(x) \cdot \boldsymbol{n}_{K, \sigma}$ on $\sigma=K \mid L$ and $\sigma \in \mathcal{E}_{K} \cap \mathcal{E}_{\text {ext }}$. This motivates the choice of the approximate Laplacian (2).

Following the continuous framework, we define the following inner product of two elements $v, w$ of the space $H_{\mathcal{D}}(\Omega)$ :

$$
\begin{aligned}
{[v, w]_{\mathcal{D}} } & =-\int_{\Omega} w(\boldsymbol{x}) \Delta_{\mathcal{D}} v(\boldsymbol{x}) \mathrm{d} \boldsymbol{x}=-\int_{\Omega} v(\boldsymbol{x}) \Delta_{\mathcal{D}} w(\boldsymbol{x}) \mathrm{d} \boldsymbol{x} \\
& =\frac{1}{2} \sum_{K \in \mathcal{M}} \sum_{L \in \mathcal{N}_{K}} \frac{\mathrm{m}_{K \mid L}}{d_{K \mid L}}\left(v_{L}-v_{K}\right)\left(w_{L}-w_{K}\right)+\sum_{K \in \mathcal{M}} \sum_{\sigma \in \mathcal{E}_{K} \cap \mathcal{E}_{\text {ext }}} \frac{\mathrm{m}_{\sigma}}{d_{K, \sigma}} v_{K} w_{K} .
\end{aligned}
$$

The corresponding norm of $w \in H_{\mathcal{D}}(\Omega)$, denoted by

$$
\|w\|_{\mathcal{D}}=\left([w, w]_{\mathcal{D}}\right)^{1 / 2},
$$

satisfies the discrete Poincaré inequality (see e.g. [9]), given by:

$$
\|w\|_{L^{2}(\Omega)} \leq \operatorname{diam}(\Omega)\|w\|_{\mathcal{D}}, \forall w \in H_{\mathcal{D}}(\Omega) .
$$

For any continuous function $\varphi$, we define the interpolation $P_{\mathcal{D}} \varphi \in H_{\mathcal{D}}(\Omega)$ by

$$
\left(P_{\mathcal{D}} \varphi\right)_{K}=\varphi\left(\boldsymbol{x}_{K}\right) \text {, for all } K \in \mathcal{M} \text {. }
$$

Similarly, for $\boldsymbol{u}=\left(u^{(i)}\right)_{i=1, \ldots, d} \in\left(H_{\mathcal{D}}(\Omega)\right)^{d}, \boldsymbol{v}=\left(v^{(i)}\right)_{i=1, \ldots, d} \in\left(H_{\mathcal{D}}(\Omega)\right)^{d}$ and $\boldsymbol{w}=$ $\left(w^{(i)}\right)_{i=1, \ldots, d} \in\left(H_{\mathcal{D}}(\Omega)\right)^{d}$, we define:

$$
\|\boldsymbol{u}\|_{\mathcal{D}}=\left(\sum_{i=1}^{d}\left[u^{(i)}, u^{(i)}\right]_{\mathcal{D}}\right)^{1 / 2}, \quad[\boldsymbol{v}, \boldsymbol{w}]_{\mathcal{D}}=\sum_{i=1}^{d}\left[v^{(i)}, w^{(i)}\right]_{\mathcal{D}}
$$

and, for all vector field $\boldsymbol{\varphi}$ given by $d$ continuous components, we define $\boldsymbol{P}_{\mathcal{D}} \boldsymbol{\varphi} \in H_{\mathcal{D}}(\Omega)^{d}$ by $\left(\boldsymbol{P}_{\mathcal{D}} \varphi\right)_{K}=\boldsymbol{\varphi}\left(\boldsymbol{x}_{K}\right)$, for all $K \in \mathcal{M}$.

Next, we wish to define a discrete divergence operator which approximates the continuous divergence term $\nabla \cdot(\bar{z} \overline{\boldsymbol{u}})$, where $\bar{z}$ is a scalar function from $\Omega$ to $\mathbb{R}$ (say a concentration or a temperature) and $\overline{\boldsymbol{u}}$ is a vector function, from $\Omega$ to $\mathbb{R}^{d}$ (typically a velocity). Again using the Gauss divergence theorem, we get that

$$
\int_{K} \operatorname{div}(\bar{z} \overline{\boldsymbol{u}})=\int_{\partial K} \bar{z} \overline{\boldsymbol{u}} \cdot \boldsymbol{n}_{K} \mathrm{~d} \gamma(\boldsymbol{x})=\sum_{\sigma \in \mathcal{E}_{K}} \int_{\sigma} \bar{z} \overline{\boldsymbol{u}} \cdot \boldsymbol{n}_{K, \sigma} \mathrm{d} \gamma(\boldsymbol{x}) .
$$

Assuming the homogeneous Dirichlet boundary condition for $\overline{\boldsymbol{u}}$, a natural (centred) finite volume approximation of the divergence of $\bar{z} \overline{\boldsymbol{u}}$ is therefore $\operatorname{div}_{\mathcal{D}}^{0}(z, \boldsymbol{u}) \in H_{\mathcal{D}}(\Omega)$, such that for all $K \in \mathcal{M}$, its constant value $\operatorname{div}_{K}^{0}(z, \boldsymbol{u})$ on $K$ is given by:

$$
\operatorname{div}_{K}^{0}(z, \boldsymbol{u})=\frac{1}{\mathrm{~m}_{K}} \sum_{L \in \mathcal{N}_{K}} \frac{\mathrm{m}_{K \mid L}}{d_{K \mid L}} \boldsymbol{n}_{K L} \cdot\left(d_{L, K \mid L} \boldsymbol{u}_{K}+d_{K, K \mid L} \boldsymbol{u}_{L}\right) \frac{z_{K}+z_{L}}{2},
$$

where $z \in H_{\mathcal{D}}(\Omega)$ and $\boldsymbol{u} \in H_{\mathcal{D}}(\Omega)^{d}$ are the sought approximations of $z$ and $\boldsymbol{u}$. However, since the approximations of the velocity and the pressure fields are collocated, we are led to define 
a modified discrete divergence operator which involves a family of stabilization parameters, denoted by $\lambda$, and the pressure field. Hence, for a velocity field $\boldsymbol{u} \in H_{\mathcal{D}}(\Omega)^{d}$, for a pressure field $p \in H_{\mathcal{D}}(\Omega)$, for a scalar field $z \in H_{\mathcal{D}}(\Omega)$, we define the discrete divergence operator $\operatorname{div}_{\mathcal{D}}(z, \boldsymbol{u}, \lambda, p) \in H_{\mathcal{D}}(\Omega)$, such that for all $K \in \mathcal{M}$, its constant value $\operatorname{div}_{K}(z, \boldsymbol{u}, \lambda, p)$ on $K$ is given by:

$$
\begin{aligned}
& \operatorname{div}_{K}(z, \boldsymbol{u}, \lambda, p)=\frac{1}{\mathrm{~m}_{K}} \sum_{L \in \mathcal{N}_{K}} \Phi_{K L}(\boldsymbol{u}, \lambda, p) \frac{z_{K}+z_{L}}{2}, \\
& \text { with } \\
& \Phi_{K L}(\boldsymbol{u}, \lambda, p)=\frac{\mathrm{m}_{K \mid L}}{d_{K \mid L}}\left(\mathbf{n}_{K L} \cdot\left(d_{L, K \mid L} \boldsymbol{u}_{K}+d_{K, K \mid L} \boldsymbol{u}_{L}\right)-\lambda_{K \mid L}\left(p_{L}-p_{K}\right)\right), \forall L \in \mathcal{N}_{K},
\end{aligned}
$$

where $\lambda=\left(\lambda_{\sigma}\right)_{\sigma \in \mathcal{E}_{\text {int }}}$ is a given family of nonnegative real numbers. Our first choice of stabilization consists in choosing $\lambda$ as

$$
\lambda_{\sigma}=\bar{\lambda} h_{\mathcal{D}}^{\alpha}, \forall \sigma \in \mathcal{E}_{\text {int }},
$$

for a given $\bar{\lambda}>0$ and $\alpha \in(0,2)$; this yields a stabilization term à la Brezzi-Pitkäranta [6]. Our second choice of stabilization consists in choosing a strictly positive value $\bar{\lambda}$ and introducing a partition of $\mathcal{M}$, denoted by $\mathcal{C}$; the elements of $\mathcal{C}$, called clusters in this paper, are subsets of $\mathcal{M}$ : examples are shown in Figures 3 and 11. We then set

$$
\begin{array}{ll}
\lambda_{K \mid L}=\bar{\lambda} & \text { if there exists } G \in \mathcal{C} \text { with }\{K, L\} \subset G, \\
\lambda_{K \mid L}=0 & \text { otherwise. }
\end{array}
$$

Several algorithms can be used for partitioning $\mathcal{M}$. One can initialize the clusters by all the control volumes neighboring some initial given control volumes as in the example presented in Figure 3, or by all control volumes having a common vertex as in the example presented in Figure 11. Then the isolated remaining control volumes are included in the cluster which already contains the greatest number of its neighbors.

Note that in (7) as well as in (6), the velocity field between the two control volumes $K$ and $L$ is interpolated in order to ensure an optimal error estimate (see [14]). For all $\boldsymbol{v} \in H_{\mathcal{D}}(\Omega)^{d}$, the approximation $\operatorname{div}_{\mathcal{D}}(\boldsymbol{v}, \boldsymbol{u}, \lambda, p)$ of $\operatorname{div}(\overline{\boldsymbol{v}} \otimes \overline{\boldsymbol{u}})$ is obtained by replacing in (7) the scalars $z_{K}$ and $z_{L}$ by the vectors $\boldsymbol{v}_{K}$ and $\boldsymbol{v}_{L}$, that is:

$$
\operatorname{div}_{K}(\boldsymbol{v}, \boldsymbol{u}, \lambda, p)=\frac{1}{\mathrm{~m}_{K}} \sum_{L \in \mathcal{N}_{K}} \Phi_{K L}(\boldsymbol{u}, \lambda, p) \frac{\boldsymbol{v}_{K}+\boldsymbol{v}_{L}}{2} .
$$

Finally, let us define a discrete gradient operator as the adjoint of the non stabilized divergence operator given in (6); for any discrete field $p \in H_{\mathcal{D}}(\Omega)$, we define its discrete gradient $\nabla_{\mathcal{D}} p \in H_{\mathcal{D}}(\Omega)^{d}:$

$$
\int_{\Omega} \nabla_{\mathcal{D}} p(\boldsymbol{x}) \cdot \boldsymbol{v}(\boldsymbol{x}) \mathrm{d} \boldsymbol{x}=-\int_{\Omega} p(\boldsymbol{x}) \operatorname{div}_{\mathcal{D}}^{0}(1, \boldsymbol{v})(\boldsymbol{x}) \mathrm{d} \boldsymbol{x}, \forall \boldsymbol{v} \in H_{\mathcal{D}}(\Omega)^{d} .
$$

This implies the following definition for the constant value $\nabla_{K} p$ of $\nabla_{\mathcal{D}} p$ in $K \in \mathcal{M}$ :

$$
\nabla_{K} p=\frac{1}{\mathrm{~m}_{K}} \sum_{L \in \mathcal{N}_{K}} \frac{d_{L, K \mid L}}{d_{K \mid L}} \mathrm{~m}_{K \mid L} \boldsymbol{n}_{K L}\left(p_{L}-p_{K}\right) .
$$


Defining $p_{\sigma}=\frac{d_{K, K \mid L}}{d_{K \mid L}} p_{K}+\frac{d_{L, K \mid L}}{d_{K \mid L}} p_{L}$ if $\sigma=K \mid L$ (note that this interpolation is not natural, contrary to that of (6) or (7)), and $p_{\sigma}=p_{K}$ if $\sigma \in \mathcal{E}_{\text {ext }} \cap \mathcal{E}_{K}$, and using the fact that $\sum_{\sigma \in \mathcal{E}_{K}} \mathrm{~m}_{\sigma} \boldsymbol{n}_{K, \sigma}=0$, one notices that $\nabla_{K} p$ may also be written

$$
\nabla_{K} p=\frac{1}{\mathrm{~m}_{K}} \sum_{\sigma \in \mathcal{E}_{K}} \mathrm{~m}_{\sigma} p_{\sigma} \boldsymbol{n}_{K, \sigma}
$$

Using the above definitions, we are now able to write the scheme for the linear Stokes equations in the next section. The extension to a nonlinear case is done in section 3 .

\subsection{The finite volume scheme in the linear case}

Consider the generalized stationary Stokes equations on $\Omega \subset \mathbb{R}^{d}$ obtained after a time discretization of the unsteady Stokes problem:

$$
\begin{cases}\eta \overline{\boldsymbol{u}}-\nu \Delta \overline{\boldsymbol{u}}+\nabla \bar{p} & =\boldsymbol{f} \\ \operatorname{div} \overline{\boldsymbol{u}} & =0\end{cases}
$$

where $\nu$ is the kinematic viscosity, $\eta$ is a frequency depending on the time scheme, $\overline{\boldsymbol{u}}=$ $\left(\bar{u}_{i}\right)_{i=1, \ldots, d}$ denotes the velocity field, $d$ is the space dimension, and $\bar{p}$ the pressure field, with the following assumptions:

$\Omega$ is a polygonal open bounded connected subset of $\mathbb{R}^{d}, d=2$ or 3

$$
\nu \in(0,+\infty), \eta \in[0,+\infty), \boldsymbol{f} \in L^{2}(\Omega)^{d} .
$$

Assuming the homogeneous Dirichlet boundary condition for $\overline{\boldsymbol{u}}$ on $\partial \Omega$, the weak formulation of (13) is given by:

$$
\left\{\begin{array}{l}
\overline{\boldsymbol{u}} \in H_{0}^{1}(\Omega)^{d}, \bar{p} \in L^{2}(\Omega) \text { with } \int_{\Omega} \bar{p}(\boldsymbol{x}) \mathrm{d} \boldsymbol{x}=0, \\
\eta \int_{\Omega} \overline{\boldsymbol{u}}(\boldsymbol{x}) \cdot \overline{\boldsymbol{v}}(\boldsymbol{x}) \mathrm{d} \boldsymbol{x}+\nu \int_{\Omega} \nabla \overline{\boldsymbol{u}}(\boldsymbol{x}): \nabla \overline{\boldsymbol{v}}(\boldsymbol{x}) \mathrm{d} \boldsymbol{x}-\int_{\Omega} \bar{p}(\boldsymbol{x}) \operatorname{div} \overline{\boldsymbol{v}}(\boldsymbol{x}) \mathrm{d} \boldsymbol{x} \\
=\int_{\Omega} \boldsymbol{f}(\boldsymbol{x}) \cdot \overline{\boldsymbol{v}}(\boldsymbol{x}) \mathrm{d} \boldsymbol{x}, \forall \overline{\boldsymbol{v}} \in H_{0}^{1}(\Omega)^{d}, \\
\operatorname{div} \overline{\boldsymbol{u}}(\boldsymbol{x})=0 \text { for a.e. } \boldsymbol{x} \in \Omega .
\end{array}\right.
$$

Let $\mathcal{D}$ be an admissible discretization of $\Omega$. For a given family of nonnegative real numbers $\lambda=\left(\lambda_{\sigma}\right)_{\sigma \in \mathcal{E}_{\text {int }}}$, we look for $(\boldsymbol{u}, p)$ such that

$$
\left\{\begin{array}{l}
(\boldsymbol{u}, p) \in H_{\mathcal{D}}(\Omega)^{d} \times H_{\mathcal{D}}(\Omega) \text { with } \int_{\Omega} p(\boldsymbol{x}) \mathrm{d} \boldsymbol{x}=0 \\
\eta \int_{\Omega} \boldsymbol{u}(\boldsymbol{x}) \cdot \boldsymbol{v}(\boldsymbol{x}) \mathrm{d} \boldsymbol{x}+\nu[\boldsymbol{u}, \boldsymbol{v}]_{\mathcal{D}}-\int_{\Omega} p(\boldsymbol{x}) \operatorname{div}_{\mathcal{D}}^{0}(1, \boldsymbol{v})(\boldsymbol{x}) \mathrm{d} \boldsymbol{x}=\int_{\Omega} \boldsymbol{f}(\boldsymbol{x}) \cdot \boldsymbol{v}(\boldsymbol{x}) \mathrm{d} \boldsymbol{x} \\
\forall \boldsymbol{v} \in H_{\mathcal{D}}(\Omega)^{d} \\
\operatorname{div}_{\mathcal{D}}(1, \boldsymbol{u}, \lambda, p)(\boldsymbol{x})=0, \text { for a.e. } x \in \Omega
\end{array}\right.
$$

System (17) is equivalent to finding the family of vectors $\left(\boldsymbol{u}_{K}\right)_{K \in \mathcal{M}} \subset \mathbb{R}^{d}$, and scalars $\left(p_{K}\right)_{K \in \mathcal{M}} \subset \mathbb{R}$ solution of the system of equations obtained by writing for each control volume 
$K$ of $\mathcal{M}$ :

$$
\left\{\begin{array}{l}
\eta \boldsymbol{u}_{K}-\nu \Delta_{K} \boldsymbol{u}+\nabla_{K} p=\frac{1}{\mathrm{~m}_{K}} \int_{K} \boldsymbol{f}(\boldsymbol{x}) \mathrm{d} \boldsymbol{x} \\
\operatorname{div}_{K}(1, \boldsymbol{u}, \lambda, p)=0
\end{array}\right.
$$

supplemented by the relation

$$
\sum_{K \in \mathcal{M}} \mathrm{m}_{K} p_{K}=0
$$

Thanks to definition (2) and to the relation (12), we see that (18) can be written in a conservative form, that indicates this scheme is indeed a finite volume scheme. We can then prove (see [13] for details) that if $(\boldsymbol{u}, p) \in H_{\mathcal{D}}(\Omega)^{d} \times H_{\mathcal{D}}(\Omega)$ is a solution to (17), then the following inequalities hold:

$$
\nu\|\boldsymbol{u}\|_{\mathcal{D}} \leq \operatorname{diam}(\Omega)\|\boldsymbol{f}\|_{\left(L^{2}(\Omega)\right)^{d}},
$$

and

$$
\frac{1}{2} \sum_{K \in \mathcal{M}} \sum_{L \in \mathcal{N}_{K}} \frac{\mathrm{m}_{K \mid L}}{d_{K \mid L}} \lambda_{K \mid L}\left(p_{L}-p_{K}\right)^{2} \leq \frac{\operatorname{diam}(\Omega)}{\nu}\|\boldsymbol{f}\|_{\left(L^{2}(\Omega)\right)^{d}}^{2} .
$$

We now give some consequences of these a priori estimates, restricting our study to the case where there exists some $\bar{\lambda}>0$ and $\alpha \in(0,2)$ such that (8) holds (note that under some geometrical assumptions on the mesh, convergence may also be proved for the stabilization by clusters, see [15] and [16]). We then get the uniqueness of a discrete solution to (17), and, using the Nečas technique, we can prove the following estimate on the pressure:

$$
\|p\|_{L^{2}(\Omega)} \leq C_{1}\|\boldsymbol{f}\|_{\left(L^{2}(\Omega)\right)^{d}},
$$

where $C_{1}$ only depends on $d, \Omega, \eta, \nu, \bar{\lambda}, \alpha$ and on any $\theta>0$ such that $\operatorname{regul}(\mathcal{D}) \geq \theta$, and not on $h_{\mathcal{D}}$.

From these estimates, and thanks to some estimates on the translates which may be obtained in a similar way as in [9], we obtain compactness properties of sequences of approximate solutions, as the mesh size tends to 0. Passing to the limit in the scheme, we then get the following convergence result [13]:

Theorem 2.1 (Convergence) Under hypotheses (14)-(15), let $(\overline{\boldsymbol{u}}, \bar{p})$ be the unique weak solution of the Stokes problem (13). Let $\bar{\lambda} \in(0,+\infty), \alpha \in(0,2)$ and $\theta>0$ be given and let $\mathcal{D}$ be an admissible discretization of $\Omega$ such that $\operatorname{regul}(\mathcal{D}) \geq \theta$ (see definitions in section 2.1). Let $\left(\boldsymbol{u}_{\mathcal{D}}, p_{\mathcal{D}}\right) \in H_{\mathcal{D}}(\Omega)^{d} \times H_{\mathcal{D}}(\Omega)$ be the unique solution to $(17)$ with $\lambda$ defined by (8). Then $\boldsymbol{u}_{\mathcal{D}}$ converges to $\overline{\boldsymbol{u}}$ in $L^{2}(\Omega)^{d}$ and $p_{\mathcal{D}}$ weakly converges to $\bar{p}$ in $L^{2}(\Omega)$ as $h_{\mathcal{D}}$ tends to 0 .

Note that the proof of the strong convergence of $p_{\mathcal{D}}$ to $\bar{p}$ is also a straightforward consequence of the error estimate which we now state (also given in [13]), under additional regularity hypotheses.

Theorem 2.2 (Error estimate) Under hypotheses (14)-(15), we assume that the weak solution $(\overline{\boldsymbol{u}}, \bar{p})$ of the Stokes problem (13) i.e. satisfying (16) is such that $(\overline{\boldsymbol{u}}, \bar{p}) \in H^{2}(\Omega)^{d} \times$ $H^{1}(\Omega)$. Let $\bar{\lambda} \in(0,+\infty)$ and $\alpha \in(0,2)$ be given, let $\mathcal{D}$ be an admissible discretization of $\Omega$ and let $\theta>0$ such that $\operatorname{regul}\left(\mathcal{D}^{(m)}\right) \geq \theta$ (see definitions in section 2.1). Let $\left(\boldsymbol{u}_{\mathcal{D}}, p_{\mathcal{D}}\right) \in$ 
$H_{\mathcal{D}}(\Omega)^{d} \times H_{\mathcal{D}}(\Omega)$ be the solution to (17) with $\lambda$ defined by (8). Then there exists $C_{2}$, which only depends on $d, \Omega, \nu, \eta$ and $\theta$ such that

$$
\begin{gathered}
\left\|\boldsymbol{u}_{\mathcal{D}}-\overline{\boldsymbol{u}}\right\|_{L^{2}(\Omega)}^{2} \leq C_{2} \epsilon\left(\bar{\lambda}, h_{\mathcal{D}}, \bar{p}, \overline{\boldsymbol{u}}\right) \\
\bar{\lambda} h_{\mathcal{D}}^{\alpha}\left|p_{\mathcal{D}}\right|_{\mathcal{D}}^{2} \leq C_{2} \epsilon\left(\bar{\lambda}, h_{\mathcal{D}}, \bar{p}, \overline{\boldsymbol{u}}\right) \\
\left\|p_{\mathcal{D}}-\bar{p}\right\|_{L^{2}(\Omega)}^{2} \leq C_{2} \epsilon\left(\bar{\lambda}, h_{\mathcal{D}}, \bar{p}, \overline{\boldsymbol{u}}\right) .
\end{gathered}
$$

where

$$
\epsilon\left(\bar{\lambda}, h_{\mathcal{D}}, \bar{p}, \overline{\boldsymbol{u}}\right)=\max \left(\bar{\lambda} h_{\mathcal{D}}^{\alpha}, \frac{h_{\mathcal{D}}^{2-\alpha}}{\bar{\lambda}}\right)\left(\|\bar{p}\|_{H^{1}(\Omega)}^{2}+\|\overline{\boldsymbol{u}}\|_{H^{2}(\Omega)}^{2}\right) .
$$

Hence, for $\alpha=1$ we get an order $1 / 2$ for the convergence of the scheme. In fact, this result is not sharp, and the numerical results show a much better order of convergence.

\section{The Navier-Stokes equations under the Boussinesq approximation}

Let us consider in this section the case of the steady equations obtained after a time discretization of the Navier-Stokes and energy equations, under the Boussinesq hypotheses

$$
\begin{array}{lll}
\eta \overline{\boldsymbol{u}}-\nu \Delta \overline{\boldsymbol{u}}+\nabla \bar{p}+\operatorname{div}(\overline{\boldsymbol{u}} \otimes \overline{\boldsymbol{u}}) & =\boldsymbol{f}+\bar{T} \boldsymbol{w} & \text { in } \Omega, \\
\operatorname{div} \overline{\boldsymbol{u}} & =0 & \text { in } \Omega, \\
\eta \bar{T}-\kappa \Delta \bar{T}+\operatorname{div}(\bar{T} \overline{\boldsymbol{u}}) & =g & \text { in } \Omega .
\end{array}
$$

with homogeneous Dirichlet boundary conditions on $\overline{\boldsymbol{u}}$ and $\bar{T}$, assumptions (14)-(15) on $\Omega, \nu$, $\eta$ and $\boldsymbol{f}$ and with $\kappa$ the thermal diffusivity, $\boldsymbol{w}$ and $g$ such that:

$$
\kappa \in(0,+\infty), \boldsymbol{w} \in \mathbb{R}^{d}, g \in L^{2}(\Omega) .
$$

Then we say that $(\overline{\boldsymbol{u}}, \bar{p}, \bar{T})$ is a weak solution of $(27)$ if

$$
\left\{\begin{array}{l}
\overline{\boldsymbol{u}} \in H_{0}^{1}(\Omega)^{d}, \bar{p} \in L^{2}(\Omega) \text { with } \int_{\Omega} \bar{p}(\boldsymbol{x}) \mathrm{d} \boldsymbol{x}=0, \bar{T} \in H_{0}^{1}(\Omega), \\
\int_{\Omega}(\eta \overline{\boldsymbol{u}}(\boldsymbol{x})+\operatorname{div}(\overline{\boldsymbol{u}} \otimes \overline{\boldsymbol{u}})(\boldsymbol{x})) \cdot \overline{\boldsymbol{v}}(\boldsymbol{x}) \mathrm{d} \boldsymbol{x}+\nu \int_{\Omega} \nabla \overline{\boldsymbol{u}}(\boldsymbol{x}): \nabla \overline{\boldsymbol{v}}(\boldsymbol{x}) \mathrm{d} \boldsymbol{x} \\
\quad-\int_{\Omega} \bar{p}(\boldsymbol{x}) \operatorname{div} \overline{\boldsymbol{v}}(\boldsymbol{x}) \mathrm{d} \boldsymbol{x}=\int_{\Omega}(\boldsymbol{f}(\boldsymbol{x})+\bar{T}(\boldsymbol{x}) \boldsymbol{w}) \cdot \overline{\boldsymbol{v}}(\boldsymbol{x}) \mathrm{d} \boldsymbol{x}, \quad \forall \overline{\boldsymbol{v}} \in H_{0}^{1}(\Omega)^{d}, \\
\int_{\Omega}(\eta \bar{T}(\boldsymbol{x})+\operatorname{div}(\bar{T} \overline{\boldsymbol{u}})(\boldsymbol{x})) \bar{S}(\boldsymbol{x}) \mathrm{d} \boldsymbol{x}+\kappa \int_{\Omega} \nabla \bar{T}(\boldsymbol{x}) \cdot \nabla \bar{S}(\boldsymbol{x}) \mathrm{d} \boldsymbol{x} \\
=\int_{\Omega} g(\boldsymbol{x}) \bar{S}(\boldsymbol{x}) \mathrm{d} \boldsymbol{x}, \quad \forall \bar{S} \in H_{0}^{1}(\Omega), \\
\operatorname{div} \overline{\boldsymbol{u}}(\boldsymbol{x})=0 \text { for a.e. } x \in \Omega .
\end{array}\right.
$$

We now give the finite volume scheme for this problem. Under hypotheses (14)-(15) and (28), let $\mathcal{D}$ be an admissible discretization of $\Omega$. For a given family of nonnegative real numbers 
$\lambda=\left(\lambda_{\sigma}\right)_{\sigma \in \mathcal{E}_{\text {int }}}$, we look for $(\boldsymbol{u}, p, T)$ such that

$$
\left\{\begin{array}{l}
(\boldsymbol{u}, p, T) \in H_{\mathcal{D}}(\Omega)^{d} \times H_{\mathcal{D}}(\Omega) \times H_{\mathcal{D}}(\Omega) \text { with } \int_{\Omega} p(\boldsymbol{x}) \mathrm{d} \boldsymbol{x}=0, \\
\nu[\boldsymbol{u}, \boldsymbol{v}]_{\mathcal{D}}+\int_{\Omega}\left(\eta \boldsymbol{u}(\boldsymbol{x})+\operatorname{div}_{\mathcal{D}}(\boldsymbol{u}, \boldsymbol{u}, \lambda, p)(\boldsymbol{x})\right) \cdot \boldsymbol{v}(\boldsymbol{x}) \mathrm{d} \boldsymbol{x} \\
-\int_{\Omega} p(\boldsymbol{x}) \operatorname{div}_{\mathcal{D}}^{0}(1, \boldsymbol{v})(\boldsymbol{x}) \mathrm{d} \boldsymbol{x}=\int_{\Omega}(\boldsymbol{f}(\boldsymbol{x})+T(\boldsymbol{x}) \boldsymbol{w}) \cdot \boldsymbol{v}(\boldsymbol{x}) \mathrm{d} \boldsymbol{x}, \forall \boldsymbol{v} \in H_{\mathcal{D}}(\Omega)^{d} \\
\operatorname{div}_{\mathcal{D}}(1, \boldsymbol{u}, \lambda, p)=0, \\
\kappa[T, S]_{\mathcal{D}}+\int_{\Omega}\left(\eta T(\boldsymbol{x})+\operatorname{div}_{\mathcal{D}}(T, \boldsymbol{u}, \lambda, p)(\boldsymbol{x})\right) S(\boldsymbol{x}) \mathrm{d} \boldsymbol{x}=\int_{\Omega} g(\boldsymbol{x}) S(\boldsymbol{x}) \mathrm{d} \boldsymbol{x}, \\
\forall S \in H_{\mathcal{D}}(\Omega) .
\end{array}\right.
$$

Note that the relation $\operatorname{div}_{\mathcal{D}}(1, \boldsymbol{u}, \lambda, p)=0$ implies

$$
\begin{gathered}
\int_{\Omega} \boldsymbol{v}(\boldsymbol{x}) \cdot \operatorname{div}_{\mathcal{D}}(\boldsymbol{u}, \boldsymbol{u}, \lambda, p)(\boldsymbol{x}) \mathrm{d} \boldsymbol{x}=-\int_{\Omega} \boldsymbol{u}(\boldsymbol{x}) \cdot \operatorname{div}_{\mathcal{D}}(\boldsymbol{v}, \boldsymbol{u}, \lambda, p)(\boldsymbol{x}) \mathrm{d} \boldsymbol{x}, \\
\int_{\Omega} S(\boldsymbol{x}) \operatorname{div}_{\mathcal{D}}(T, \boldsymbol{u}, \lambda, p)(\boldsymbol{x}) \mathrm{d} \boldsymbol{x}=-\int_{\Omega} T(\boldsymbol{x}) \operatorname{div}_{\mathcal{D}}(S, \boldsymbol{u}, \lambda, p)(\boldsymbol{x}) \mathrm{d} \boldsymbol{x} .
\end{gathered}
$$

which shows that the discrete versions of the operators behave as the continuous ones. Again, system (30) is equivalent to finding the family of vectors $\left(\boldsymbol{u}_{K}\right)_{K \in \mathcal{M}} \subset \mathbb{R}^{d}$, and scalars $\left(p_{K}, T_{K}\right)_{K \in \mathcal{M}} \subset \mathbb{R}$ such that, for each control volume $K$ of $\mathcal{M}$ :

$$
\left\{\begin{array}{l}
\eta \boldsymbol{u}_{K}-\nu \Delta_{K} \boldsymbol{u}+\operatorname{div}_{K}(\boldsymbol{u}, \boldsymbol{u}, \lambda, p)+\nabla_{K} p=\frac{1}{\mathrm{~m}_{K}} \int_{K} \boldsymbol{f}(\boldsymbol{x}) \mathrm{d} \boldsymbol{x}+T_{K} \boldsymbol{w}, \\
\operatorname{div}_{K}(1, \boldsymbol{u}, \lambda, p)=0 \\
\eta T_{K}-\kappa \Delta_{K} T+\operatorname{div}_{K}(T, \boldsymbol{u}, \lambda, p)=\frac{1}{\mathrm{~m}_{K}} \int_{K} g(\boldsymbol{x}) \mathrm{d} \boldsymbol{x},
\end{array}\right.
$$

supplemented by the relation:

$$
\sum_{K \in \mathcal{M}} \mathrm{m}_{K} p_{K}=0
$$

One should notice that, thanks to (31), one has two ways of implementing the scheme (32). These two ways are not equivalent from the point of view of the convergence properties of the numerical algorithms described in Section 4.1.

Theorem 3.1 (Convergence of the scheme) Under hypotheses (14)-(15) and (28), let $\left(\mathcal{D}^{(m)}\right)_{m \in \mathbb{N}}$ be a sequence of admissible discretizations of $\Omega$ such that $h_{\mathcal{D}^{m}}$ tends to 0 as $m \rightarrow \infty$ and such that there exists $\theta>0$ with $\operatorname{regul}\left(\mathcal{D}^{(m)}\right) \geq \theta$, for all $m \in \mathbb{N}$ (see definitions in section 2.1). Let $\bar{\lambda} \in(0,+\infty)$ and $\alpha \in(0,2)$ be given. Then, for all $m \in \mathbb{N}$, there exists at least one solution to (30) with $\mathcal{D}=\mathcal{D}^{(m)}$ and $\lambda=\lambda^{(m)}$ defined by (8), that we denote $\left(\boldsymbol{u}^{(m)}, p^{(m)}, T^{(m)}\right) \in\left(H_{\mathcal{D}^{(m)}}(\Omega)\right)^{d} \times H_{\mathcal{D}^{(m)}}(\Omega) \times H_{\mathcal{D}^{(m)}}(\Omega)$. Moreover, there exists $(\overline{\boldsymbol{u}}, \bar{p}, \bar{T})$ satisfying (29) and a subsequence of $\left(\mathcal{D}^{(m)}\right)_{m \in \mathbb{N}}$, again denoted $\left(\mathcal{D}^{(m)}\right)_{m \in \mathbb{N}}$, such that the corresponding subsequence of solutions $\left(\boldsymbol{u}^{(m)}, T^{(m)}\right)_{m \in \mathbb{N}}$ converges to $(\overline{\boldsymbol{u}}, \bar{T})$ in $L^{2}(\Omega)$ and $\left(p^{(m)}\right)_{m \in \mathbb{N}}$ weakly converges to $\bar{p}$ in $L^{2}(\Omega)$.

Proof Several keypoints of the proof of this convergence result may be found in [13], where we showed the convergence of the scheme for the isothermal Navier-Stokes equations. The main 
differences with [13] are the fact that we have to handle the additional convection-diffusion equation on the temperature, and that we do not use the Bernoulli pressure thanks to the definition (7) of the discrete divergence, which includes the stabilization pressure term. Let us then notice that, thanks to (31), we get

$$
\int_{\Omega} \boldsymbol{u}(\boldsymbol{x}) \cdot \operatorname{div}_{\mathcal{D}}(\boldsymbol{u}, \boldsymbol{u}, \lambda, p)(\boldsymbol{x}) \mathrm{d} \boldsymbol{x}=0 \text { and } \int_{\Omega} T(\boldsymbol{x}) \operatorname{div}_{\mathcal{D}}(T, \boldsymbol{u}, \lambda, p)(\boldsymbol{x}) \mathrm{d} \boldsymbol{x}=0 .
$$

Hence, taking $\boldsymbol{u}=\boldsymbol{v}$ in the first equation of (30) and $S=T$ in the last one, we get the estimate

$$
\kappa\|T\|_{\mathcal{D}} \leq \operatorname{diam}(\Omega)\|g\|_{L^{2}(\Omega)},
$$

and therefore

$$
\nu\|\boldsymbol{u}\|_{\mathcal{D}} \leq \operatorname{diam}(\Omega)\left(\|\boldsymbol{f}\|_{L^{2}(\Omega)^{d}}+|\boldsymbol{w}| \frac{\operatorname{diam}(\Omega)}{\kappa}\|g\|_{L^{2}(\Omega)}\right),
$$

and, thanks to (8),

$$
\begin{aligned}
& \bar{\lambda} h_{\mathcal{D}}^{\alpha} \frac{1}{2} \sum_{K \in \mathcal{M}} \sum_{L \in \mathcal{N}_{K}} \frac{\mathrm{m}_{K \mid L}}{d_{K \mid L}}\left(p_{L}-p_{K}\right)^{2} \\
& \leq \frac{\operatorname{diam}(\Omega)}{\nu}\left(\|\boldsymbol{f}\|_{\left(L^{2}(\Omega)\right)^{d}}+|\boldsymbol{w}| \frac{\operatorname{diam}(\Omega)}{\kappa}\|g\|_{L^{2}(\Omega)}\right)^{2} .
\end{aligned}
$$

We then deduce the existence of $C_{1}$, only depending on $d, \Omega, \eta, \nu, \bar{\lambda}, \kappa, \boldsymbol{w}$ and $\theta$, and not on $h_{\mathcal{D}}$, such that

$$
\|p\|_{L^{2}(\Omega)} \leq C_{1}\left(\|\boldsymbol{f}\|_{L^{2}(\Omega)^{d}}+\|g\|_{L^{2}(\Omega)}+\left(\|\boldsymbol{f}\|_{L^{2}(\Omega)^{d}}+\|g\|_{L^{2}(\Omega)}\right)^{2}\right)
$$

in a similar way as in [13]. These estimates are sufficient to allow the application of the topological degree method (see theorem 4.3 of [13]). Hence, for all $m \in \mathbb{N}$, we prove the existence of at least one solution $\left(\boldsymbol{u}^{(m)}, p^{(m)}, T^{(m)}\right) \in H_{\mathcal{D}^{(m)}}(\Omega)^{d} \times H_{\mathcal{D}^{(m)}}(\Omega) \times H_{\mathcal{D}^{(m)}}(\Omega)$ to (30) with $\mathcal{D}=\mathcal{D}^{(m)}$ and $\lambda=\lambda^{(m)}$ defined by (8). These estimates also prove the existence of $\overline{\boldsymbol{u}} \in H_{0}^{1}(\Omega)^{d}$, $\bar{p} \in L^{2}(\Omega)$ with $\int_{\Omega} \bar{p}(\boldsymbol{x}) \mathrm{d} \boldsymbol{x}=0$, of $\bar{T} \in H_{0}^{1}(\Omega)$, and of a subsequence of $\left(\mathcal{D}^{(m)}\right)_{m \in \mathbb{N}}$, again denoted $\left(\mathcal{D}^{(m)}\right)_{m \in \mathbb{N}}$, such that $\left(\boldsymbol{u}^{(m)}\right)_{m \in \mathbb{N}}$ converges in $L^{2}(\Omega)$ to $\overline{\boldsymbol{u}},\left(p^{(m)}\right)_{m \in \mathbb{N}}$ weakly converges in $L^{2}(\Omega)$ to $\bar{p}$ and $\left(T^{(m)}\right)_{m \in \mathbb{N}}$ converges in $L^{2}(\Omega)$ to $\bar{T}$. It remains now to prove that $(\overline{\boldsymbol{u}}, \bar{p}, \bar{T})$ satisfies (29). Note that $\operatorname{div} \overline{\boldsymbol{u}}(\boldsymbol{x})=0$ for a.e. $\boldsymbol{x} \in \Omega$ is obtained in the same way as in [13]. The only new points to check concern the right hand side $\boldsymbol{f}+T \boldsymbol{w}$ instead of $\boldsymbol{f}$, the additional equation and the nonlinear terms. Since $\left(T^{(m)}\right)_{m \in \mathbb{N}}$ converges in $L^{2}(\Omega)$ to $\bar{T}$, the convergence to $\boldsymbol{f}+\bar{T} \boldsymbol{w}$ of its discrete counterpart is clear. Let $\varphi \in C_{c}^{\infty}(\Omega)^{d}$ be given. Let us prove that $\int_{\Omega} \boldsymbol{P}_{\mathcal{D}^{(m)}} \boldsymbol{\varphi}(\boldsymbol{x}) \cdot \operatorname{div}_{\mathcal{D}}\left(\boldsymbol{u}^{(m)}, \boldsymbol{u}^{(m)}, \lambda^{(m)}, p^{(m)}\right)(\boldsymbol{x}) \mathrm{d} \boldsymbol{x}$ converges to $\int_{\Omega} \boldsymbol{\varphi}(\boldsymbol{x}) \cdot \boldsymbol{d i v}(\overline{\boldsymbol{u}} \otimes \overline{\boldsymbol{u}})(\boldsymbol{x}) \mathrm{d} \boldsymbol{x}$ as $m \rightarrow \infty$.

For a given discretisation $\mathcal{D}$ and for $(\boldsymbol{u}, p) \in H_{\mathcal{D}}(\Omega)^{d} \times H_{\mathcal{D}}(\Omega)$, defining $\lambda$ by (8), let us write $\int_{\Omega} \boldsymbol{P}_{\mathcal{D}} \boldsymbol{\varphi}(\boldsymbol{x}) \cdot \operatorname{div}_{\mathcal{D}}(\boldsymbol{u}, \boldsymbol{u}, \lambda, p)(\boldsymbol{x}) \mathrm{d} \boldsymbol{x}=T_{1}(\mathcal{D}, \boldsymbol{u}, p)+T_{2}(\mathcal{D}, \boldsymbol{u}, p)$ with

$$
\begin{aligned}
T_{1}(\mathcal{D}, \boldsymbol{u}, p) & =\frac{1}{2} \sum_{K \in \mathcal{M}} \sum_{L \in \mathcal{N}_{K}} \Phi_{K L}(\boldsymbol{u}, 0,0)\left(\frac{\boldsymbol{u}_{L}+\boldsymbol{u}_{K}}{2} \cdot\left(\boldsymbol{\varphi}\left(\boldsymbol{x}_{K}\right)-\boldsymbol{\varphi}\left(\boldsymbol{x}_{L}\right)\right)\right), \\
T_{2}(\mathcal{D}, \boldsymbol{u}, p) & =\int_{\Omega} \boldsymbol{u}(\boldsymbol{x}) \cdot \boldsymbol{G}_{\mathcal{D}}(p, \boldsymbol{\varphi})(\boldsymbol{x}) \mathrm{d} \boldsymbol{x}
\end{aligned}
$$


where we use the notation $\Phi_{K L}$ defined in (7), and where the function $\boldsymbol{G}_{\mathcal{D}}(p, \boldsymbol{\varphi}): \Omega \rightarrow \mathbb{R}^{d}$ is defined, for all $K \in \mathcal{M}$ and $\sigma \in \mathcal{E}_{K}$, by the constant value $\boldsymbol{G}_{K, \sigma}(p, \boldsymbol{\varphi})$ in the cone $D_{K, \sigma}$ with basis $\sigma$ and vertex $\boldsymbol{x}_{K}$, such that

$$
\boldsymbol{G}_{K, K \mid L}(p, \boldsymbol{\varphi})=\frac{1}{2} \frac{\bar{\lambda} h_{\mathcal{D}}^{\alpha}}{\mathrm{m}\left(D_{K, K \mid L}\right)} \frac{\mathrm{m}_{K \mid L}}{d_{K \mid L}}\left(p_{L}-p_{K}\right)\left(\boldsymbol{\varphi}\left(\boldsymbol{x}_{L}\right)-\boldsymbol{\varphi}\left(\boldsymbol{x}_{K}\right)\right), \forall L \in \mathcal{N}_{K},
$$

and

$$
\boldsymbol{G}_{K, \sigma}(p, \boldsymbol{\varphi})=0, \forall \sigma \in \mathcal{E}_{K} \cap \mathcal{E}_{\text {ext }} .
$$

We remark that, thanks to $(36), \boldsymbol{G}_{\mathcal{D}^{(m)}}\left(p^{(m)}, \boldsymbol{\varphi}\right)$ converges to $\mathbf{0}$ in $L^{2}(\Omega)^{d}$ as as $m \rightarrow+\infty$. Hence we get that

$$
\lim _{m \rightarrow \infty} T_{2}\left(\mathcal{D}^{(m)}, \boldsymbol{u}^{(m)}, p^{(m)}\right)=0 .
$$

In the same way as in [13], we have

$$
\lim _{m \rightarrow \infty} T_{1}\left(\mathcal{D}^{(m)}, \boldsymbol{u}^{(m)}, p^{(m)}\right)=\int_{\Omega} \boldsymbol{\varphi}(\boldsymbol{x}) \cdot \operatorname{div}(\overline{\boldsymbol{u}} \otimes \overline{\boldsymbol{u}})(\boldsymbol{x}) \mathrm{d} \boldsymbol{x} .
$$

Hence, the expression $\int_{\Omega} \boldsymbol{P}_{\mathcal{D}^{(m)}} \boldsymbol{\varphi}(\boldsymbol{x}) \cdot \operatorname{div}_{\mathcal{D}}\left(\boldsymbol{u}^{(m)}, \boldsymbol{u}^{(m)}, \lambda^{(m)}, p^{(m)}\right)(\boldsymbol{x}) \mathrm{d} \boldsymbol{x}$ converges to $\int_{\Omega} \boldsymbol{\varphi}(\boldsymbol{x})$. $\operatorname{div}(\overline{\boldsymbol{u}} \otimes \overline{\boldsymbol{u}})(\boldsymbol{x})) \mathrm{d} \boldsymbol{x}$ as $m \rightarrow \infty$. For a given function $\varphi \in C_{c}^{\infty}(\Omega)$, the convergence proof of $\int_{\Omega} P_{\mathcal{D}^{(m)}} \varphi(\boldsymbol{x}) \operatorname{div}_{\mathcal{D}}\left(T^{(m)}, \boldsymbol{u}^{(m)}, \lambda^{(m)}, p^{(m)}\right)(\boldsymbol{x}) \mathrm{d} \boldsymbol{x}$ to $\left.\int_{\Omega} \varphi(\boldsymbol{x}) \operatorname{div}(\bar{T} \overline{\boldsymbol{u}})(\boldsymbol{x})\right) \mathrm{d} \boldsymbol{x}$ as $m \rightarrow \infty$ follows similar arguments. The convergence of $\left[T^{(m)}, P_{\mathcal{D}^{(m)}} \varphi\right]_{\mathcal{D}^{(m)}}$ to $\int_{\Omega} \nabla \bar{T} \cdot \nabla \varphi$ is proven in Proposition 2.4 of [13], and the convergence study of the corresponding right hand side is straightforward. This completes the proof of the theorem.

Remark 1. Note that it is possible to prove a convergence theorem, similar to Theorem 3.1, in the case of nonhomogeneous Dirichlet boundary and $g=0$. We then consider the upstream approximation of $\operatorname{div}(\bar{T} \overline{\boldsymbol{u}})$, given by

$$
\operatorname{div}_{K}^{u p s}(T, \boldsymbol{u}, \lambda, p)=\frac{1}{\mathrm{~m}_{K}}\left(\sum_{L \in \mathcal{N}_{K}}\left(\Phi_{K L}(\boldsymbol{u}, \lambda, p)^{+} T_{K}-\Phi_{K L}(\boldsymbol{u}, \lambda, p)^{-} T_{L}\right)\right),
$$

where $\Phi_{K L}(\boldsymbol{u}, \lambda, p)$ is defined in (7), and where, for all $s \in \mathbb{R}$, we set $s^{+}=\max (s, 0)$ and $s^{-}=\max (-s, 0)$. In this case, it is possible to derive an $L^{\infty}$ estimate on $T$, which allows for similar inequalities to (35) and (36), and then it is easy to conclude the convergence proof, following the methods used in [9] in the case of the approximation by the finite volume method of a nonhomogeneous Dirichlet elliptic problem. Note that the convergence of the scheme, keeping the centered approximation (7) of $\operatorname{div}_{K}(T, \boldsymbol{u}, \lambda, p)$ instead using $\operatorname{div}_{K}^{\text {ups }}(T, \boldsymbol{u}, \lambda, p)$, remains an open problem, because the lack of a bound on the velocities prevents from getting the stability of the resulting centred convection diffusion scheme. Nevertheless, the centred approximation remains stable in many cases, including the numerical tests given in section 4.5.

\section{Numerical results}

\subsection{Practical implementation}

Let us first comment the nature of the different problems to be solved. The scheme (18) is a coupled linear problem with a symmetric matrix, but the scheme (32) is a coupled nonlinear 
problem, the Jacobian matrix of which is nonsymmetric (taking the opposite of the fluid mass conservation equation). Our numerical choices are as follows:

1. The nonlinear problem (32) is solved by a coupled under-relaxed Newton iteration method (in order to avoid large variations of the unknown in one iteration which could lead to the divergence of the method). This implies that at each iteration, a linear system involving the Jacobian matrix of the full nonlinear system, must be solved, with 4 unknowns per control volume in 2D and 5 in 3D. Several advantages are gained from this procedure: there is no approximate Poisson problem to solve in order to compute the pressure (it is well known that such a procedure induces the appearance of boundary layers), the method is completely systematic and the programming easy; furthermore steady problems may be solved directly, without using artificial transient problems.

2. The coupled linear systems are solved in our prototype, using either a direct solver (Gaussian elimination without pivoting, for small tests) or a GMRES or BiCGSTAB method for large ones with a incomplete LU preconditioning. These methods have been shown to be efficient in all the test cases which are presented in this paper.

Another advantage of the numerical method that we have used is that the accuracy which is requested in the linear system resolution can be linked with the residual at each Newton iteration, which avoids wasting time in precise linear resolution when the Newton iteration is still far for convergence.

\subsection{Study of the stabilization methods}

We implemented the two stabilization techniques (8) and (9) for the steady and transient Navier-Stokes equations with or without the energy equation [7]. Let us first show on an example the importance of introducing a stabilization term in the numerical scheme. We consider the case $\Omega=(0,1) \times(0,1), \eta=0, \nu=1$ and $g=0$ (it corresponds to an isothermal case), and $\boldsymbol{f}$ chosen such that $(27)$ holds with $\bar{T}(x, y)=0, \bar{u}^{(1)}(x, y)=-\partial_{y} \Phi(x, y)$ and $\bar{u}^{(2)}(x, y)=\partial_{x} \Phi(x, y)$, computed from the potential $\Phi(x, y)=600[x(1-x) y(1-y)]^{2}$, the pressure being given by $\bar{p}(x, y)=100\left(x^{2}+y^{2}-\frac{2}{3}\right)$, for all $(x, y) \in \Omega$. We then obtain that the numerical solution obtained for the velocity is accurate whatever the method and the magnitude of stabilization. On the contrary, it is clear from Figure 2 that the pressure field suffers from severe oscillations when $\bar{\lambda}=0$ or is close to 0 ; these results are obtained using the cluster stabilization (9) (the clusters are shown on Figure 3), but a similar behaviour on the pressure is obtained with the Brezzi Pitkäranta stabilization.

Let us compare the two stabilization terms respectively given by (8) and (9). An advantage of (8) is that it leads to an easier mathematical analysis of the convergence of the scheme, but a drawback is that it yields some redistribution of the fluid mass over the whole domain. Moreover, in order to obtain convergence, one needs to let the stabilization parameter tend to 0 with the size of the mesh $h_{\mathcal{D}}$ (hence the expression of the stabilization term in (8)). On the contrary, in the stabilization term given by (9), there is no need to let $\bar{\lambda}$ tend to 0 with the size of the mesh, which means that the presence of a finite stabilization does not decrease the quality of the approximation. Let us show this property on the backward facing step example at Reynolds number 800 (the complete data are presented, for example, in [8]). We show in Figure 4 the computed isovalue lines of the horizontal component of the velocity 

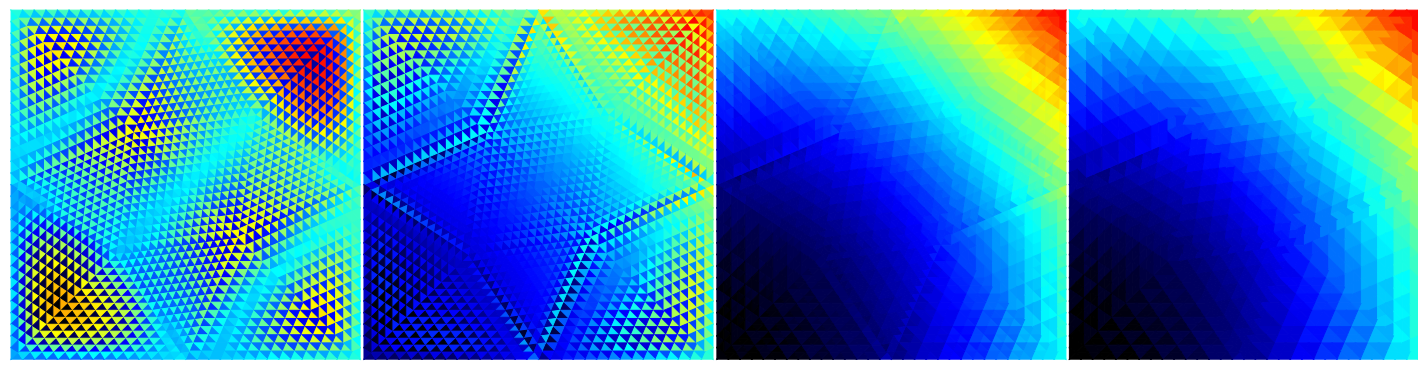

Figure 2. Pressure field for different values of $\bar{\lambda}$ (from the left to the right: $0,10^{-6}, 10^{-4}, 10^{-2}$ )
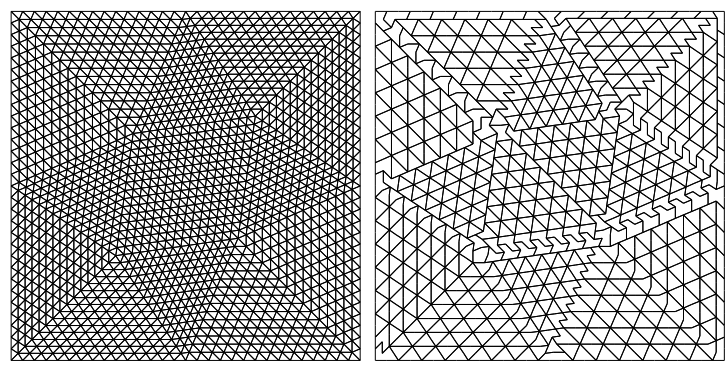

Figure 3. Mesh (left) and clusters (right)

$u^{(1)}$, obtained for a mesh of 6300 cells. Roughly speaking, the isovalue lines $u^{(1)}=0$ (dashed in Figure 4) separates the two recirculation vortices; the determination of this isoline therefore allows the evaluation of the reattachment lengths of both vortices. We then study the effect of the stabilization term on these reattachment lengths. In order to do so, we consider a given mesh and decrease the stabilization term. This effect is shown in Figure 5, where we draw the values of both reattachment lengths computed with the same mesh, as a function of the value of the "weight" of the stabilization, that is the global value of $\bar{\lambda} h_{\mathcal{D}}^{\alpha}$ in (8), and $\bar{\lambda}$ in (9). We see that, as the stabilization value increases, the reattachment lengths obtained with the BrezziPitkäranta stabilization decrease much more than those given by the cluster stabilization. This shows that, in the case of problems requesting a high stabilization level, the numerical results expected using the cluster stabilization remains more accurate. The adaptation of the convergence proofs to the cluster stabilization is the object of recent works [15], [16].

\subsection{Numerical confirmation of the convergence study}

Let us first emphasize that the order of convergence of a numerical scheme must be distinguished from the consistency order, obtained by introducing in the numerical scheme an analytical solution taken in some points: we noticed above that for example, we do not require that the approximate gradient of pressure be consistent. Indeed, the definition (11) is not consistent in the general case and we only require that it is the transpose of the discrete divergence approximate. Moreover, as mentioned above, the orders of convergence that were mathematically proven (see Theorem 2.2) are hopefully much lower that the observed numerical 


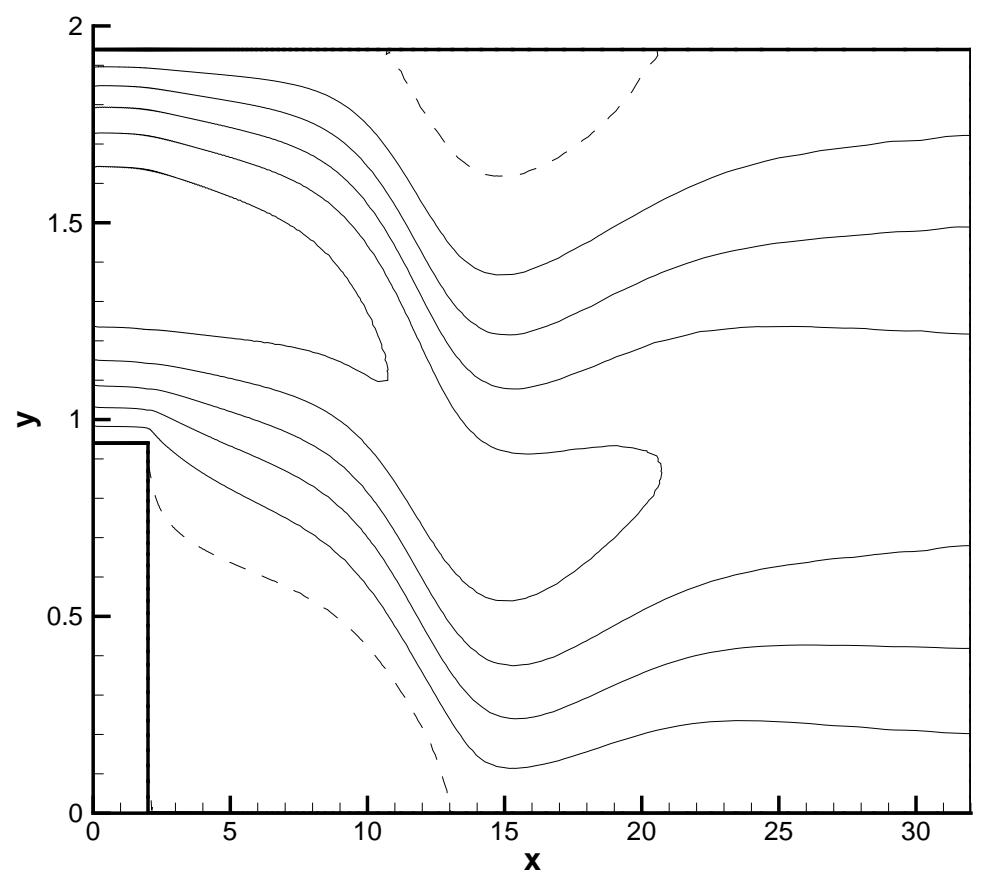

Figure 4. Isovalue lines of $u^{(1)}$ for the backward facing step (the step is visible at the lower left part of the figure) with $\operatorname{Re}=800$. The dashed lines correspond to the isovalue $u^{(1)}=0$ line.

orders of convergence.

Indeed, let us first consider the well-known transient Green-Taylor analytical solution (isothermal case), in the square domain $\Omega=(-0.5,0.5) \times(-0.5,0.5)$, given by

$$
\begin{aligned}
\bar{u}^{(1)}(x, y, t) & =-\cos (\pi x) \sin (\pi y) \exp \left(-2 \pi^{2} t / \mathrm{Re}\right) \\
\bar{u}^{(2)}(x, y, t) & =\sin (\pi x) \cos (\pi y) \exp \left(-2 \pi^{2} t / \mathrm{Re}\right) \\
\bar{p}(x, y, t) & =-\frac{\cos (2 \pi x)+\cos (2 \pi y)}{4} \exp \left(-4 \pi^{2} t / \mathrm{Re}\right),
\end{aligned}
$$

where Re is the Reynolds number. The initial and boundary conditions are deduced from the exact solution. We consider the case $\mathrm{Re}=10$, and we compare the approximate solutions given by the collocated scheme using the cluster stabilization (9), discretized by the Crank-Nicholson method, with the analytical ones at time $t=0.3$, for rectangular meshes with respectively 400 , 1600,6400 and 25600 control volumes, keeping the time step equal to 0.001 . We then plot in Figure 6 the $L^{2}$ norms of the errors $e(\boldsymbol{u})$ and $e(p)$ between the computational and the analytical solution, for these different meshes. We observe that the order of convergence, given by the slope of fitted lines on these curves, is close to 2 for the velocity, and 1 for the pressure. We study in a similar way the error as a function of the time steps, on a $160 \times 160$ rectangular mesh. The $L^{2}$ norms of the errors $e(\boldsymbol{u})$ and $e(p)$ are plotted in Figure 7 , showing similar order of convergence as in space. 


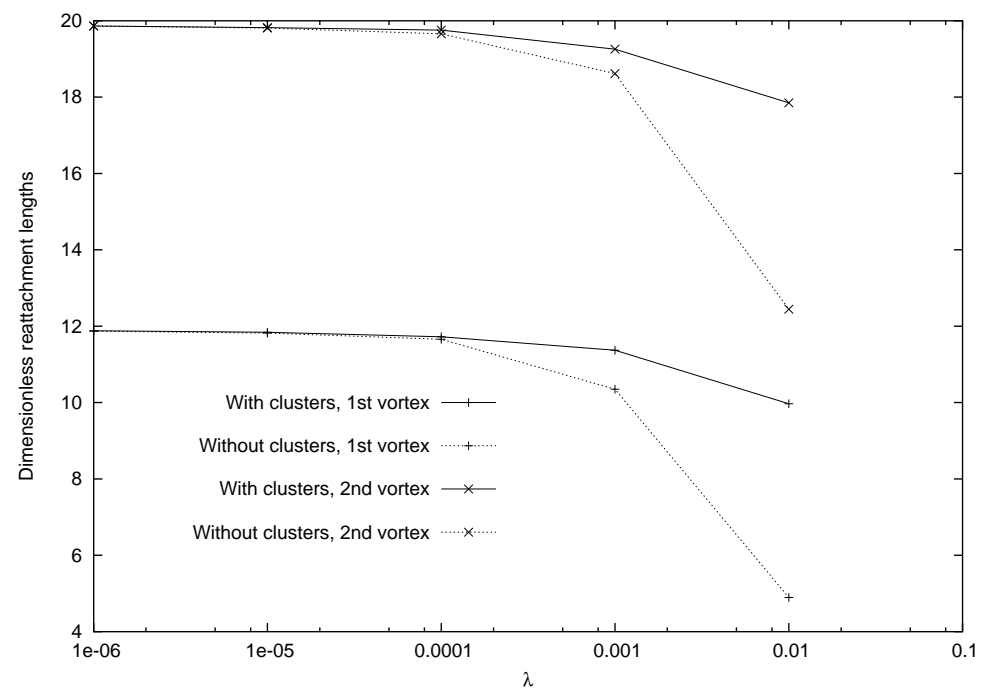

Figure 5. Reattachment lengths for different stabilization parameters. The dashed lines correspond to the Brezzi-Pitkäranta stabilization, the continuous ones to the cluster stabilization.
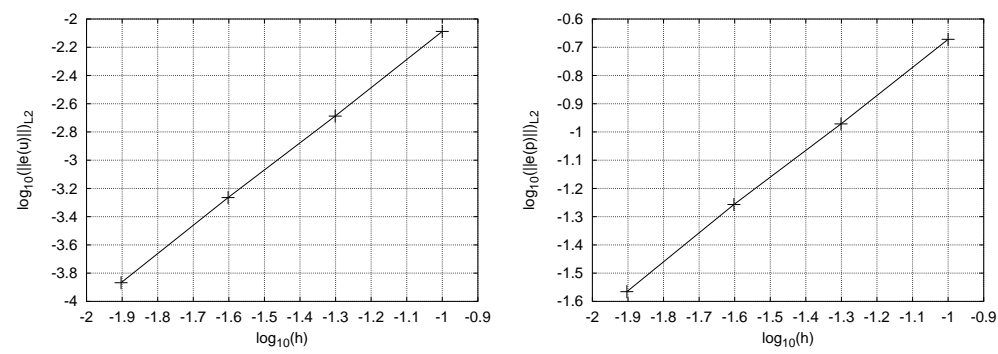

Figure 6. $L^{2}$ norm of error (left: velocity, right: pressure) for different space steps
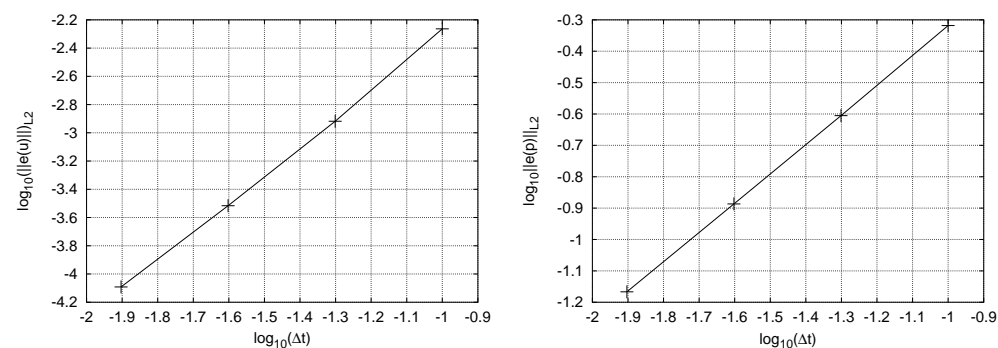

Figure $7 . L^{2}$ norm of error (left: velocity, right: pressure) for different time steps

Since there is no straightforward analytical solution with null right-hand-side in the case of non isothermal problem, we considered a steady problem $(\eta=0)$ where a divergence free

Int. J. Numer. Meth. Fluids 2006; 00:0-0 
analytical solution is arbitrarily chosen, and the right-hand-sides $f$ and $g$ are the residual of the continuous Boussinesq and energy equations without source terms. Hence we define the scalar potential $\psi$ as in [32], by

$$
\psi(x, y)=\sin ^{2}(\pi x) \sin ^{2}(\pi y),
$$

the temperature and the pressure fields are respectively given by

$$
\begin{aligned}
\bar{T}(x, y) & =\sin ^{2}(\pi x) \sin ^{2}(\pi y) \\
\bar{p}(x, y) & =\sin ^{2}(\pi x) \sin ^{2}(\pi y)
\end{aligned}
$$

and the velocity field is given by

$$
\begin{array}{r}
\bar{u}^{(1)}(x, y)=\frac{\partial \Psi}{\partial y}=2 \pi \cos (\pi y) \sin (\pi y) \sin ^{2}(\pi x) \\
\bar{u}^{(2)}(x, y)=-\frac{\partial \Psi}{\partial x}=-2 \pi \cos (\pi x) \sin (\pi x) \sin ^{2}(\pi y) .
\end{array}
$$

Choosing the cluster stabilization (9), we set $\bar{\lambda}=0.001, \nu=\kappa=1, \boldsymbol{w}$ is the unit vertical vertor pointing upward. We proceed in the same way as we did for the Green-Taylor analytical solution, computing the order of convergence for variable space steps. We found an order 2 convergence for the velocity, the temperature, and an order 1 for the pressure in the case of rectangular meshes or Delaunay meshes (see Figure 8 for example of such a mesh).

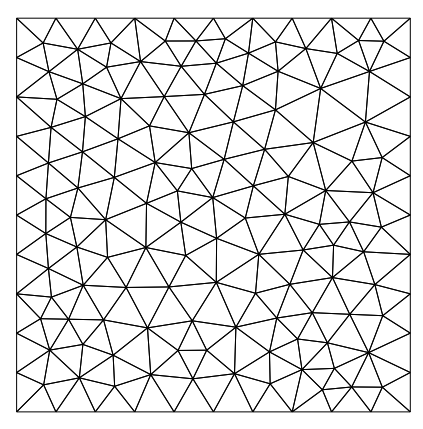

Figure 8. Example of a Delaunay mesh

\subsection{Comparison with other methods}

Let us first again consider the same backward facing step problem as the one used in Section 4.2 for the study of the effects of the stabilization method. We apply the collocated scheme, using a mesh with 109050 triangles. In table I, we provide the first and second reattachment lengths in the case $\mathrm{Re}=800$ given in the literature, together with ours. We observe that our results show an excellent agreement with that obtained by other methods.

Let us now turn to the 3D lid driven cavity test case. The considered domain is the unit cube, and we consider two values for the Reynolds number $(R e=400$ and $R e=3200)$. We compare the profiles of some components of the velocity along median lines with that given in [31] with 97336 control volumes (finite volume method on unstructured meshes). We use 


\begin{tabular}{|c||c|c|}
\hline & 1st vortex & 2nd vortex \\
\hline Armaly et al. (experimental results) [1] & 14.2 & 20.0 \\
Chiang et al. [8] & 12.3 & 20.8 \\
Kim \& Moin [25] & 12.0 & \\
Kaiktsis et al. $[24]$ & 11.9 & \\
Centred staggered scheme [12] & 13.3 & 20.6 \\
Collocated scheme & 12.0 & 19.9 \\
\hline
\end{tabular}

Table I. First and second reattachment lengths for $\mathrm{Re}=800$

a simple $36 \times 36 \times 36$ parallelepipedic mesh with refined sides along the boundaries of the domain. The stabilization parameter is taken equal to $10^{-5}$. We observe in Figure 9 that the results obtained by the collocated scheme and those which are given in the reference [31] are very close.
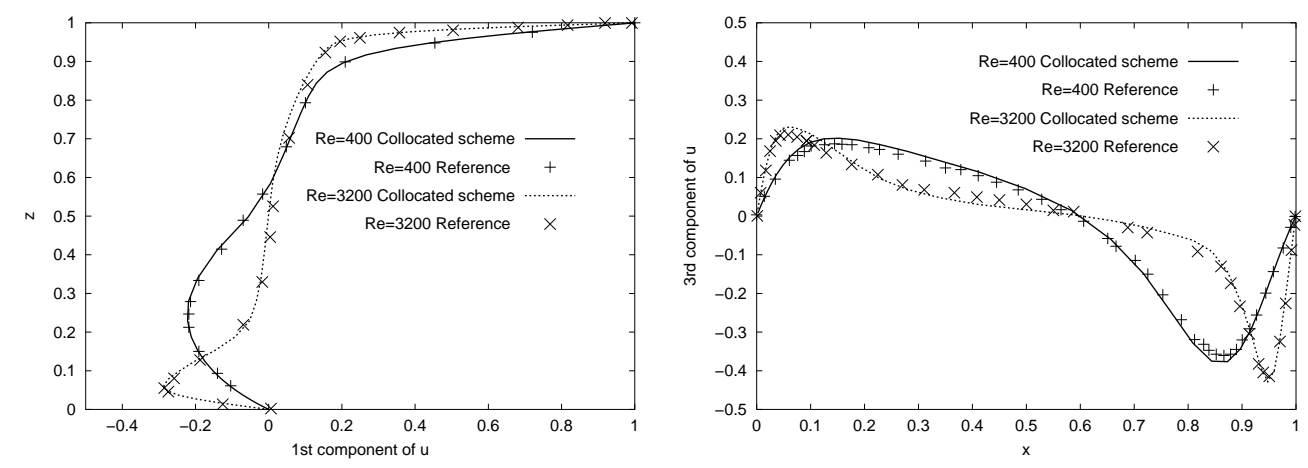

Figure 9. 3D Lid driven cavity

\subsection{Natural convection between two cylinders}

We consider the natural convective flows between two horizontal cylinders where the surface of the inner cylinder is heated. Such a configuration is, for instance, a model for thermal storage systems. An important industrial problem is therefore to control the heat transfer, either to enhance or to reduce it. Major published works have considered fluid flows and heat transfer between concentric cylinders [29]. A simple way to modify the thermal performances is to change the geometry and for instance by introducing an eccentricity in the annular space.

The geometrical characteristics of the cavity are defined by the inner $\mathcal{C}_{i}$ and outer $\mathcal{C}_{o}$ cylinders of radii $r_{i}^{*}$ and $r_{o}^{*}$, by the eccentricity $\epsilon^{*}$ and by the eccentricity angle $\phi_{\epsilon}$ (Fig. 10). In our study, uniform temperatures $T^{*}\left(\mathcal{C}_{i}\right)=T_{i}^{*}$ and $T^{*}\left(\mathcal{C}_{o}\right)=T_{o}^{*}$ are imposed on the no-slip cylinder walls (which means homogeneous Dirichlet boundary conditions for the velocity). The flow is assumed two-dimensional, steady, laminar and incompressible with constant physical properties except for the density when formulating the buoyancy effect. We then scale the lengths by the annular gap $d^{*}=r_{o}^{*}-r_{i}^{*}$, the velocity components by the ratio between the thermal diffusivity and $d^{*}$, we introduce the dimensionless difference temperature $\bar{T}=\left(T^{*}-T_{r}^{*}\right) /\left(T_{i}^{*}-T_{o}^{*}\right)$ 


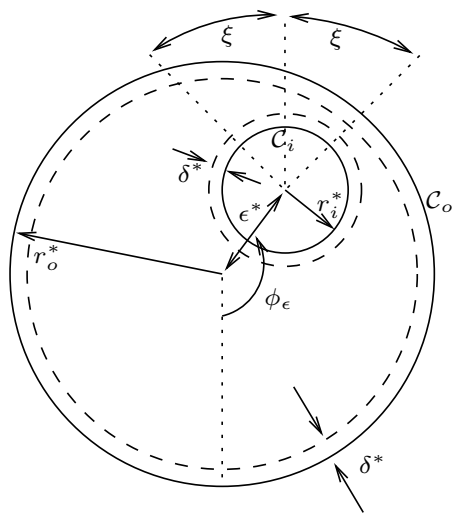

Figure 10. Geometry.

where $T_{r}^{*}=\left(T_{i}^{*}+T_{o}^{*}\right) / 2$ is the reference temperature. The dimensionless Navier-Stokes and energy equations are then given by (27) in which $\boldsymbol{f}=\mathbf{0}, \eta=0, g=0, \nu=\operatorname{Pr}, \kappa=1$ and $\boldsymbol{w}=\operatorname{Ra} \operatorname{Pr} \boldsymbol{e}^{(2)}$, where Ra and Pr are the Rayleigh and Prandtl numbers and $\boldsymbol{e}^{(2)}$ is the upward vertical unit vector. The boundary conditions for the temperature are then nonhomogeneous Dirichlet boundary conditions $\left(\bar{T}=1 / 2\right.$ on $\mathcal{C}_{i}$ and $\bar{T}=-1 / 2$ on $\left.\mathcal{C}_{o}\right)$. We denote the radius ratio by $R=r_{o}^{*} / r_{i}^{*}$, the dimensionless eccentricity by $\epsilon=\epsilon^{*} / d^{*}$ and the eccentricity angle by $\phi_{\epsilon}$.

Voronoï tessellations are used to mesh the annular space. This choice meets the definition of an admissible mesh, since the straight line joining the centers of two adjacent cells is orthogonal to their common edge (see section 2.1). Since the cell centers are mainly located at the vertices of equilateral triangles, most of the resulting Voronoï cells have hexagonal shapes. To adjust the mesh to the natural convective flows, cells with a smaller hexagonal size were used along the cylinder walls, in a layer of thickness $\delta=\delta^{*} / d^{*}$, and in an angular sector $2 \xi$ around the inner cylinder where a thermal plume and higher velocity occur (Fig. 10). The cells in the boundary layer and in the angular sector are twice smaller than the cells which define the basic hexagonal cell size. An enlargement of such a mesh is presented in figure 11a.

We use the cluster stabilization method (9), initializing the clusters by control volumes having one common vertex. Figure 11b illustrates the partition into clusters of the mesh shown in Figure 11a.

The heat transfer is measured by the mean Nusselt number at the inner cylinder

$$
\overline{N u}_{i}=\frac{1}{2 \pi r_{i}} \int_{\mathcal{C}_{i}} \nabla \bar{T}(\boldsymbol{x}) \cdot \boldsymbol{n}_{\partial \Omega}(\boldsymbol{x}) \mathrm{d} s(\boldsymbol{x}),
$$

where $r_{i}=r_{i}^{*} / d^{*}$ and $\boldsymbol{n}_{\partial \Omega}(\boldsymbol{x})$ is the normal outward unit vector at point $\boldsymbol{x} \in \mathcal{C}_{i}$. For coaxial cylinders and moderate Rayleigh number values, the flow field consists of two-dimensional crescent-shape cells, symmetrical with respect to the vertical straight line containing the centers of the cylinders. When the eccentricity is non zero and $\phi_{\epsilon} \neq 0^{\circ}, \phi_{\epsilon} \neq 180^{\circ}$, the vertical symmetry of annular space is broken and a fluid flow occurs around the inner cylinder. This flow rate is computed by $\psi_{\mathcal{C}_{i}}$ with $\psi$ the stream function defined by $u^{(1)}=\frac{\partial \psi}{\partial y}, u^{(2)}=-\frac{\partial \psi}{\partial x}$ and $\psi_{\mathcal{C}_{o}}=0$. 


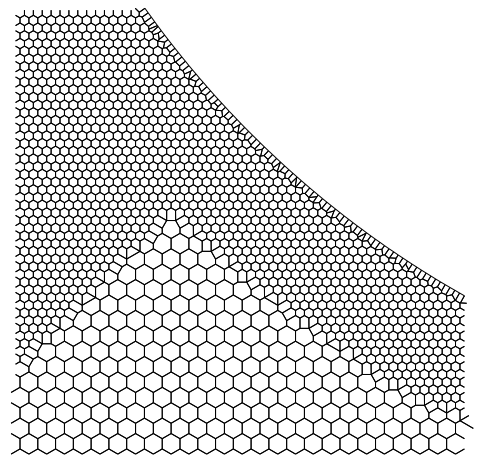

(a) Mesh

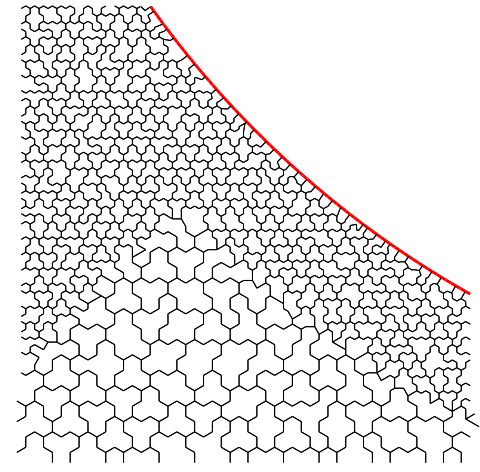

(b) clusters

Figure 11. Voronoï tessellations based on hexagonal cells and partition of the computational domain into clusters.

The effects of the eccentricity and eccentricity angle are studied for a radius ratio $R=2$, a Rayleigh number value $\mathrm{Ra}=10^{4}$ and a Prandtl number value $\mathrm{Pr}=0.7$. The angular sector and the thickness of the boundary layer, where the cell size is half the basic hexagonal cell size, are equal to $2 \xi=270^{\circ}$ and $\delta=0.2$. The basic hexagonal cell size 0.03 is classically adopted for the simulations.

In figures 12-14 are drawn the isotherms as a function of $\phi_{\epsilon}$, for $\epsilon=0,0.5$ and

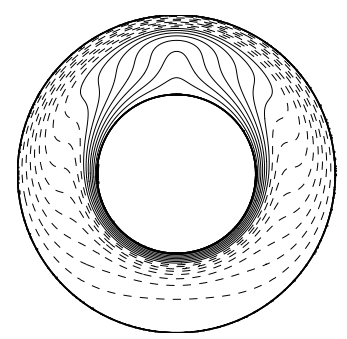

Figure 12. Isotherms of the steady solutions, $\epsilon=0$.

0.95. Continuous and dashed lines correspond to positive and negative temperature values respectively. As foreseen, the isotherms are no longer symmetric when $\phi_{\epsilon} \neq 0^{\circ}$ or $\neq 180^{\circ}$ and $\epsilon>0$. For $\phi_{\epsilon} \leq 90^{\circ}$, a thermal plume develops above the hot cylinder. On the other hand, the temperature field is rather convected from the lateral region of the inner cylinder when this latter one is located in the upper region of the outer cylinder, namely for $\phi_{\epsilon}>90^{\circ}$. When the cylinders are close, the Rayleigh number based on the smallest gap between the two walls is small, and a conduction thermal regime is therefore locally expected. Figure 15 illustrates the mean Nusselt number at the inner cylinder, $\overline{N u}_{i}$, as a function of $\phi_{\epsilon}$ and $\epsilon$. The heat 

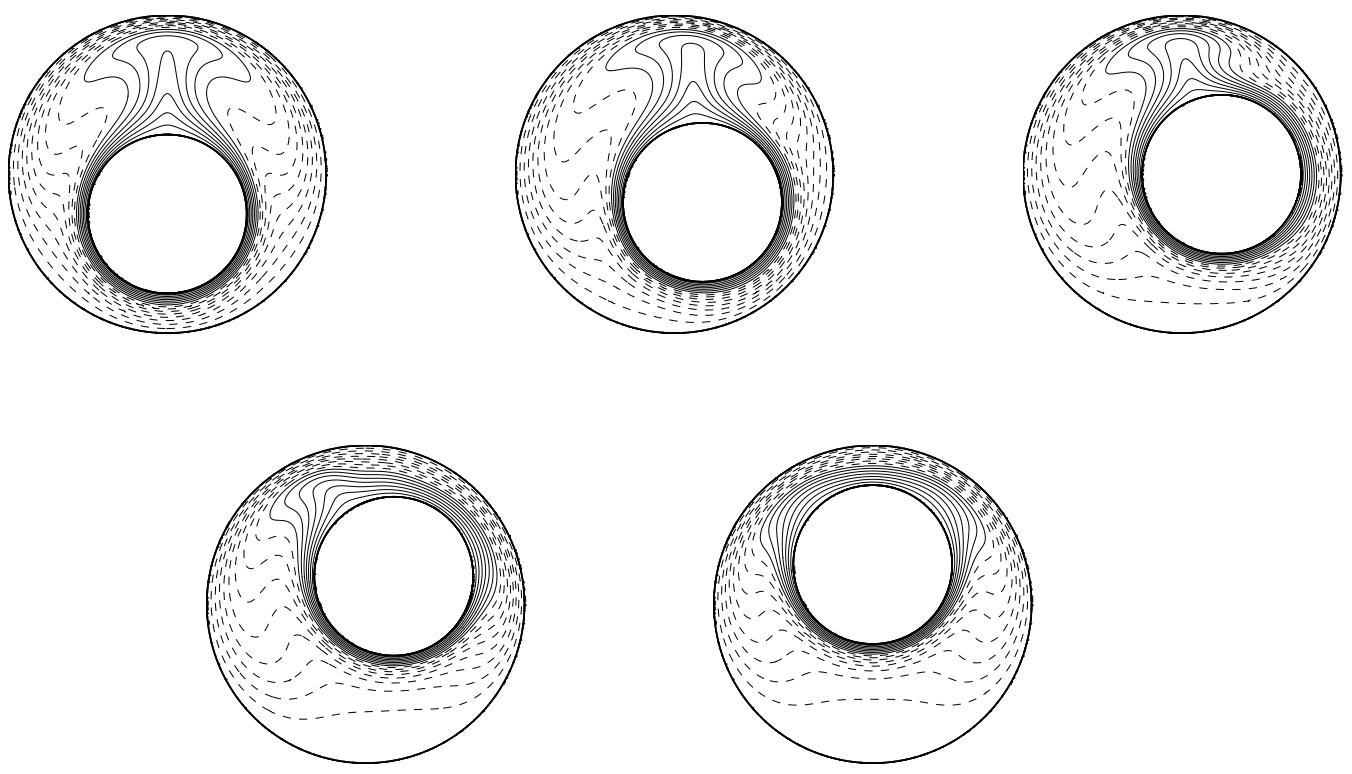

Figure 13. Isotherms of the steady solutions, $\epsilon=0.5$ and $\phi_{\epsilon}=0^{\circ}, 45^{\circ}, 90^{\circ}, 135^{\circ}, 180^{\circ}$.

transfer is weakly affected by the eccentricity angle and the eccentricity if $\epsilon<0.5$. Conversely, the mean Nusselt number is doubled between $\epsilon=0.5$ and $\epsilon=0.95$. The symmetry breaking of the geometry with respect to the vertical line containing the center of the outer cylinder generates a fluid flow circulation around the inner cylinder for which the dimensionless flow rate is measured by $\psi_{\mathcal{C}_{i}}$ (Fig. 16). The circulation is maximal for large eccentricities and when the inner cylinder is in the upper region of the outer cylinder. It is however clear that if both cylinders are too close, the fluid flows with difficulty around the hot cylinder and even cannot pass when they are side by side. In this limit case, the regularity of the boundary of the domain decreases, the boundary condition is no longer given by the trace of a regular function and the convergence of the scheme is far from being proven (the distance between the two cylinders should be discretized by a sufficient number layers of control volumes, which is maybe not the case in our computations near the limit case). As it can be observed in figures 15 and 16, some values seem to exhibit spurious variations as a function of $\phi_{\epsilon}$ and $\epsilon$. For example $\psi_{\mathcal{C}_{i}}$ decreases sharply in the vicinity of $\phi_{\epsilon} \approx 140^{\circ}$ and $\epsilon \approx 0.5$. One could also wonder whether this behavior might be explained by the occurrence of bifurcations, and a stability analysis of the steady flow should be conducted to confirm the appearance of transitions in the fluid flow. 

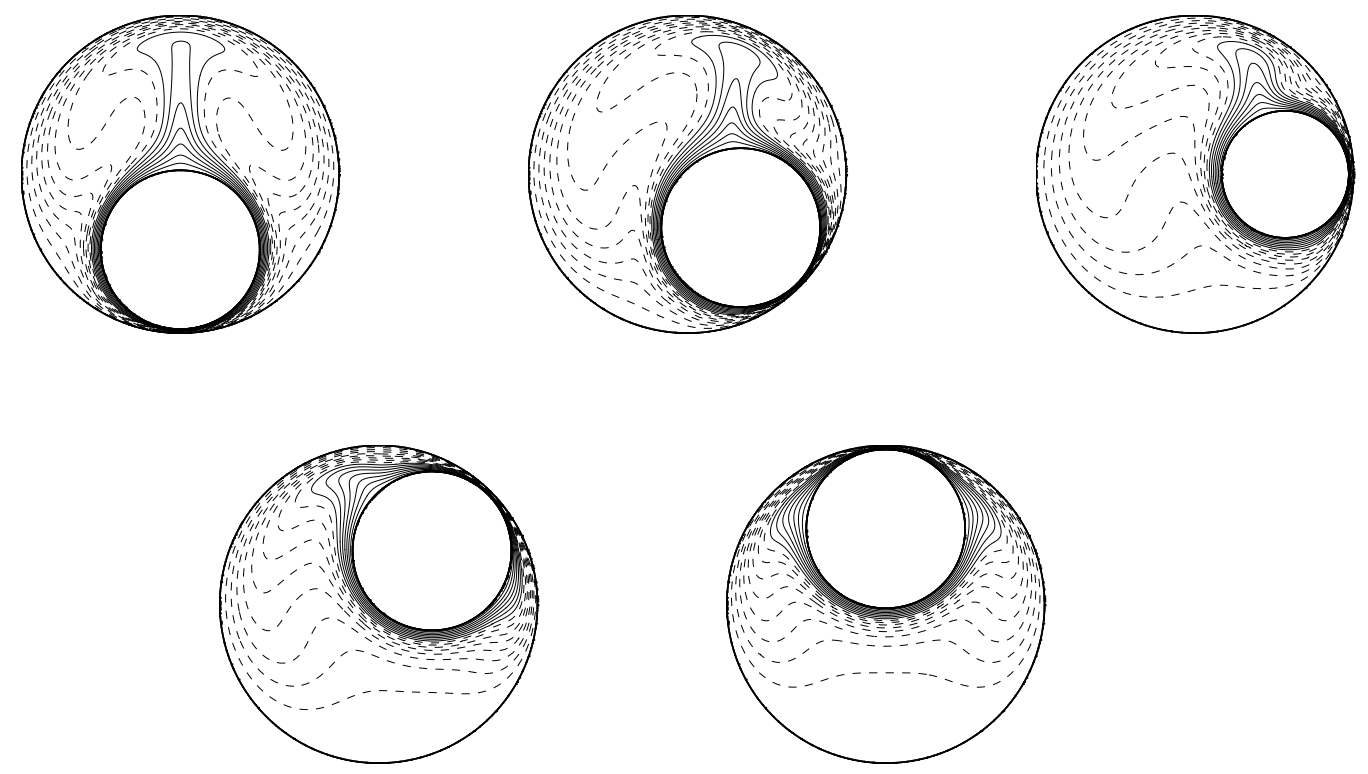

Figure 14. Isotherms of the steady solutions, $\epsilon=0.95$ and $\phi_{\epsilon}=0^{\circ}, 45^{\circ}, 90^{\circ}, 135^{\circ}, 180^{\circ}$.

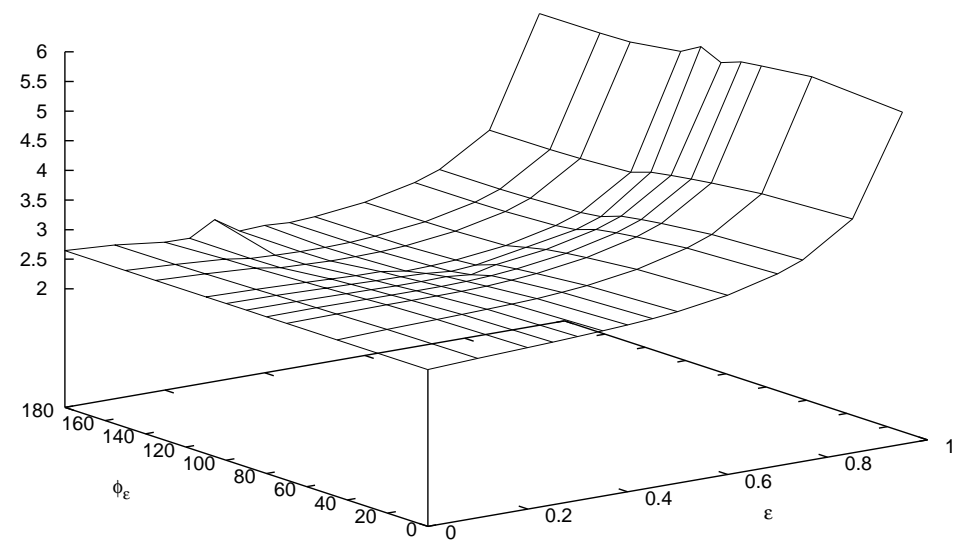

Figure 15. Mean Nusselt number at the inner cylinder, $\overline{N u}_{i}$ as a function of $\epsilon$ and $\phi_{\epsilon}$. 


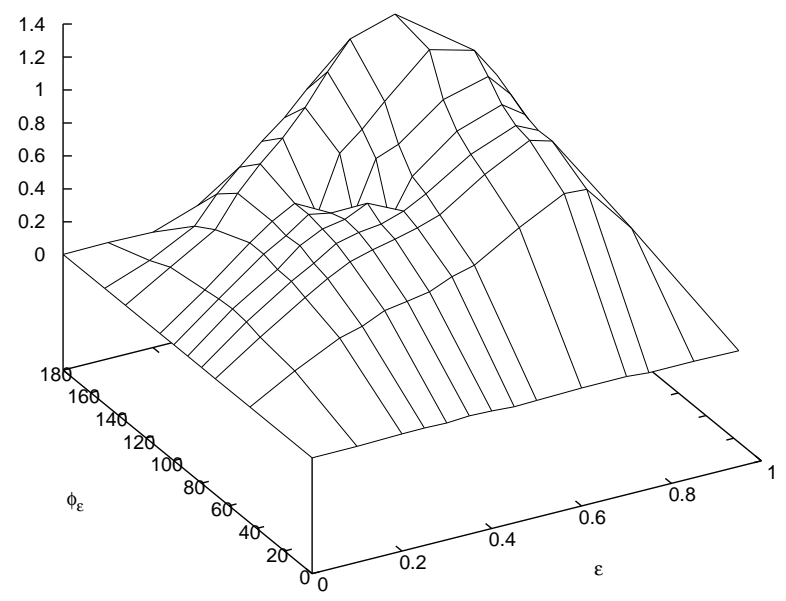

Figure 16. Dimensionless flow rate around the inner cylinder, $\psi_{\mathcal{C}_{i}}$ as a function of $\epsilon$ and $\phi_{\epsilon}$.

\section{Conclusions and perspectives}

Collocated schemes for the incompressible Navier-Stokes equations were analyzed and implemented on unstructured meshes. The collocated scheme, for which the proof of the convergence of the scheme has been completed in some cases, can handle some cases with a complex geometry, as the problem of natural convection between two cylinders with eccentricity, using unstructured meshes such as Voronoï tesselations or triangular meshes. However, the Brezzi-Pitkäranta stabilization method was found to be more sensitive to high Reynolds numbers on tests such as the backward step. The cluster stabilization was then implemented and improved dramatically the stability of the collocated scheme (in fact, clustering leads to large scale grids for the pressure). Work is in progress concerning the convergence analysis of the scheme with clustering, and of the viscous tensor term in the compressible case. The case of non constant viscosity also needs to be addressed.

\section{REFERENCES}

1. B.F. Armaly, F. Durst, J.C.F. Pereira and B. Schonung. Experimental and theoretical investigation of backward-facing step flow. J. Fluid Mech., 127:473-496, 1983.

2. S.W. Armfield, Ellipticity, accuracy and convergence of the discrete Navier-Stokes equations,. J. Comp. Phys.., 114:176-184, 1994.

3. Ph. Blanc, Convergence of a finite volume scheme on a MAC mesh for the Stokes problem with right hand side in $H^{-1}$, proceedings of FVCA4, Hermès Penton-Science, F. Benkhaldoun, D. Ouazar and S. Raghay eds., 2005, 133-142.

4. Ph. Blanc, R. Eymard and R. Herbin, A staggered finite volume scheme on general meshes for the generalized Stokes problem in two space dimensions. Int. J. Finite Volumes, 2005, http://www. latp.univmrs.fr/IJFV/

5. S. Boivin, F. Cayré and J.M. Hérard, A finite volume method to solve the Navier-Stokes equations for incompressible flows on unstructured meshes. Int. J. Therm. Sci., 38:806-825, 2000. 
6. F. Brezzi and J. Pitkäranta, On the stabilization of finite element approximations of the Stokes equations. Efficient solutions of elliptic systems, 11-19, Kiel, 1984, Notes Numer. Fluid Mech., Vieweg, Braunschweig, 10, 1984.

7. E. Chénier, O Touazi and R. Eymard, Numerical results using a collocated finite-volume scheme on unstructured grids for incompressible flows. Num. Heat Transfer, part B, 49:259-276, 2006.

8. T.P. Chiang, T.W.H. Sheu and S.F. Tsai, Topological Flow Structures in Backward-Facing Step Channels. Computers and Fluids, 26:321-337, 1997.

9. R. Eymard, T. Gallouët and R. Herbin, Finite Volume Methods, Handbook of Numerical Analysis, 7:7131020. Edited by P.G. Ciarlet and J.L. Lions (North Holland),2000.

10. R. Eymard and R. Herbin, A cell-centered finite volume scheme on general meshes for the Stokes equations in two dimensions. CRAS, Mathématiques, 337(2):125-128, 2003.

11. R. Eymard and R. Herbin, A finite volume scheme on general meshes for the steady Navier-Stokes equations in two space dimensions, Proceedings of ENUMATH03, Prague, August 2003.

12. R. Eymard and R. Herbin, A staggered finite volume scheme on general meshes for the Navier-Stokes equations in two space dimensions. Int. J. Finite Volumes, 2005, http://www.latp.univ-mrs.fr/IJFV/

13. R. Eymard, R. Herbin and J-C.Latché, Convergence analysis of a collocated finite volume scheme for the incompressible Navier-Stokes equations on general 2 or 3D meshes, SIAM Journal on Numerical Analysis, 45(1):1-36, 2007.

14. R. Eymard, R. Herbin and J-C.Latché, On a stabilized collocated Finite Volume scheme for the Stokes problem. M2AN, 40(3):501-528,2006.

15. R. Eymard, R. Herbin and J-C.Latché, Numerical analysis of a clustered collocated finite volume scheme for the incompressible Stokes Navier-Stokes equations on general 2 or 3D meshes, submitted, 2006, (see web archive: http://hal.archives-ouvertes.fr/)

16. R. Eymard, R. Herbin, J.C. Latché and B. Piar, On the stability of colocated clustered finite volume simplicial discretizations for the 2D Stokes problem, accepted for publication in Calcolo, 2007. (see also web archive: http://hal.archives-ouvertes.fr/)

17. V. Girault and P.-A. Raviart, Finite element methods for the Navier-Stokes equations: Theory and algorithms, Springer, Berlin, 1986.

18. R. Glowinski, Numerical Methods for fluids (Part3), Handbook of Numerical Analysis, Vol. IX. Edited by P.G. Ciarlet and J.L. Lions (North Holland), 2003.

19. M.D. Gunzburger, Finite element methods for viscous incompressible flows, A guide to thoery, practice, and algorithms, Computer Science and Scientific Computing, Academic Press 1989.

20. M.D. Gunzburger and R.A Nicolaïdes Incompressible computational fluid dynamics, Cambridge University Press, 1993.

21. F.H. Harlow and J.E. Welch, Numerical calculation of time dependent viscous incompressible flow of fluids with free surface. Phys. Fluids, 8:2182-2189, 1965.

22. R. Herbin, An error estimate for a finite volume scheme for a diffusion-convection problem on a triangular mesh. Num. Meth. P.D.E., 11:165-173, 1995.

23. R. Herbin, Unstructured cell centred schemes for the Navier-Stokes equations, Proceedings of the fourth symposium on finite volumes for complex applications, F. Benkhaldoun, D. Ouazar and S. Raghay eds., Hermès-Penton Science, 2005, 51-62.

24. L. Kaiktsis, G.E.M Karniadakis and S.A. Orszag. Onset of three-dimensionality, equilibria, and early transition in flow over a backward-facing step. J. Fluid Mech., 231:501-528, 1991.

25. J. Kim and P. Moin. Application of a fractionnal-step method to incompressible Navier-Stokes equations. J. Comput. Phys., 59:308-323, 1985.

26. D. Kwak and C. Kiris and C.S. Kim, Computers and Fluids, 35:283-299, 2005.

27. R.A. Nicolaïdes, Analysis and Convergence of the MAC Scheme I. The Linear Problem. SIAM J. Numer. Anal., 29:1579-1591, 1992.

28. S.V. Patankar, Numerical Heat Transfer and Fluid Flow, Series in Computational Methods in Mechanics and Thermal Sciences, Minkowycz and Sparrow Eds. (Mc Graw Hill), 1980.

29. G. Petrone, E. Chénier and G. Lauriat, Stability of free convection in air-filled horizontal annuli: Influence of the radius ratio. Int. Comm. Heat Mass Transfer, 47:3889-3907, 2004.

30. R. Peyret and T. Taylor, Computational methods for fluid flow, Springer, New-York, 1993.

31. C.H. Tai, Y. Zhao and K.M. Liew. Parallel computation of unsteady three-dimensional incompressible viscous flow using an unstructured multigrid method. Comput. \& Structures, 82:2425-2436, 2004.

32. O. Touazi. Etude numérique d'un schéma de volumes finis sur des maillages non structurés pour des écoulements de fluide visqueux à densité constante ou faiblement variable, Thèse de l'université de Marnela-Vallée, France, 2006. 